\title{
A Cosmological Model without Singularity Based on RW Metric (1)
}

\author{
Shihao Chen 1,2 \\ ${ }^{1}$ Yue-Hong Institute for Advanced Study, Yunnan University, Kunming, China \\ ${ }^{2}$ Institute of Theoretical Physics, Northeast Normal University, Changchun, China \\ Email: shchen@nenu.edu.cn \\ Received 25 December 2013; revised 23 January 2014; accepted 30 January 2014 \\ Copyright (C) 2014 by author and Scientific Research Publishing Inc. \\ This work is licensed under the Creative Commons Attribution International License (CC BY). \\ http://creativecommons.org/licenses/by/4.0/ \\ c) (i) Open Access
}

\begin{abstract}
A new conjecture is proposed that there are two sorts of matter called s-matter and $v$-matter which are symmetric, whose masses are positive, but whose gravitational masses are opposite to each other. Based on the conjecture and the $S U_{S}(5) \times S U_{V}(5)$ gauge group, a cosmological model has been constructed and the following inferences have been derived. There are two sorts of symmetry breaking called $V$-breaking and $S$-breaking. In the $V$-breaking, $S U_{V}(5)$ breaks finally to $S U_{V}(3) \times$ $U_{V}(1)$ so that $v$-particles get their masses and form $v$-atoms and $v$-galaxies etc., while $S U_{S}(5)$ still holds so that $s$-fermions and $s$-gauge bosons are massless and form $S U_{s}(5)$ color-singlets. There is no interaction among the $S U_{S}(5)$ color-singlets except gravitation so that they distribute loosely in space, cannot be observed, and cause space to expand with an acceleration. Evolution of the universe is explained. There is no space-time singularity. There are the highest temperature and the least scale in the universe. It is impossible that the Plank temperature and length are arrived. A formula is obtained which describes the relation between a luminous distance and its redshift. A huge void is not empty, and is equivalent to a huge concave lens. The densities of hydrogen in the huge voids must be much less than that predicted by the conventional theory. The gravitation between two galaxies whose distance is long enough will be less than that predicted by the conventional theory. A black hole with its big enough mass will transform into a white hole.
\end{abstract}

\section{Keywords}

Cosmology: Theories; (Cosmology:) Early Universe; (Cosmology:) Inflation; (Cosmology:) Cosmological Parameters; Galaxies: Distances and Reshifts

\section{Introduction}

In view of the fact that the space-time singularity and the cosmological constant issues are not solved in the 
frame of the conventional theory up to now. We suggest two conjectures to solve the issues. Based on the conjectures, we construct a cosmological model. Based on this model, we solve the two issues, explain the evolution of the universe, primordial nucleosynthesis, cosmic microwave background radiation $(C M B R)$, and give three new predictions.

As is now well known, there is space-time singularity under certain conditions [1]. "These conditions fall into three categories. First, there is the requirement that gravity shall be attractive. Secondly, there is the requirement that there is enough matter present in some region to prevent anything escaping from that region. The third requirement is that there should be no causality violations" [1]. There must be space-time singularity in the conventional theory, because the conditions can be satisfied.

There should be no space-time singularity in physics, hence this problem must be solved. But it is not solved satisfactorily up to now.

In order to solve the space-time singularity problem, Ref. [2] [3] had assumed that there exists a fundamental length, i.e., the Planck length, $l_{P} \sim\left(G \hbar c^{-3}\right)^{1 / 2}=1.6 \times 10^{35} \mathrm{~m}$. There is no curvature corresponding to scale $l<l_{p}$. Based on this, they proposed the limiting curvature hypothesis. Thereby they had proved that all isotropic cosmological solutions are nonsingular. We find that its conclusion is included in the hypothesis. On the other hand, the model does not explain the expansion of the universe with an acceleration and cannot solve the cosmological constant problem.

The Planck length $l_{p}$, time $t_{P} \sim\left(G \hbar c^{-5}\right)^{1 / 2}=5.4 \times 10^{-44} \mathrm{~s}$ and temperature $T_{P} \sim\left(\hbar c^{5} / G\right)^{1 / 2} / k=1.4 \times 10^{32} \mathrm{~K}$ reveal that the quantum theory and the general relativity are not self-consistent. Relativity is a theory of continuous space-time geometry. But the presence of $l_{P}, t_{P}$ and $T_{P}$ makes it virtually to come to a not continuous space-time structure. According to the conventional theory, only the quantum theory of gravity can solve the problem of not self-consistency. This is not true. According to the present model, there is no singularity of space-time, and there are the highest temperature $T_{\max } \ll T_{P}$ and the least scale $R>0$ (see later). Consequently, it is impossible that $l_{P}, t_{P}$ and $T_{P}$ are arrived, because the transformation of the $S$-breaking and $V$-breaking from one to another at the highest temperature $T_{\max }$. Thus, the quantum theory and the general relativity can be self-consistent, although the gravitational field is not quantized.

Recent astronomical observations show that the universe expanded with a deceleration earlier, while it is expanding with an acceleration now [4]-[6]. This implies that there is dark energy. Among the total energy density of the universe, 73 percent is dark energy [4]-[6]. What is dark energy? Many possibilities have been suggested. One interpretation adopts the effective cosmological constant $\lambda_{\text {eff }}=\lambda+\rho_{g \text {,vac }}$, where $\lambda$ and $\rho_{\text {g,vac }}$ are the Einstein cosmological constant and the gravitational mass density of the vacuum state, respectively. The subscript "g" denotes the physical quantities relating to gravity in the following. According to the equivalence principle, $\rho_{g, v a c}=\rho_{v a c}, \rho_{v a c}$ is the energy density of the vacuum state. Hence $\lambda_{\text {eff }}$ may be written as $\lambda+\rho_{v a c}$. $\lambda_{\text {eff }}$ cannot be derived from basic theories [7] [8], and $\rho_{\text {vac }} \ggg \lambda_{\text {eff }}$. Hence the interpretation is un- satisfactory. Alternatively, dark energy is associated with the dynamics of scalar field $\phi(t)$ which is uniform in space [9]-[11]. This is a seesaw cosmology [12]. Thus, problem about the universe expansion with an acceleration is still open to the public.

That $\rho_{g, v a c}=\rho_{v a c} \ggg \lambda_{\text {eff }}$ originates from the conventional quantum field theory and the equivalent principle. Both $\rho_{g, v a c} \ggg \lambda_{\text {eff }}$ and the singularity issues imply that the conventional theory is incomplete. In some super- symmetric models, $\rho_{v a c}=0$ can be obtained. But this is not a necessary result of the supersymmetric quantum field theory. On the other hand, supersymmetric theory lacks of experiment bases. In contrast with the super- symmetric quantum field theory, $\rho_{\text {vac }}=0$ is a necessary result of our quantum field theory without divergence [13]-[15]. In this theory, there is no divergence of loop corrections as well, and dark matter which can form dark galaxies is predicted [16] [17].

In fact, $\rho_{v a c}=0$ is not a necessary condition of $\rho_{g, v a c}=0$. We will see that although $\rho_{v a c}$ is divergent according to the conventional quantum field theory, we have still $\rho_{g \text {,vac }}=0$ based on the present model.

Huge voids in the cosmos have been observed [18]. Such a model in which hot dark matter (e.g. neutrinos) is dominant can explain the phenomenon. However, it cannot explain the structure with middle and small scales. Hence this is an open problem as well.

We consider that all important existing forms of matter (including dark matter and dark energy) have appeared. Hence these basic problems should be solved. As mentioned above, we have constructed a quantum 
field theory without divergence which predicts that there must be dark matter. We can construct a cosmological model which can solve the space-time singularity and cosmological constant issues and explain the evolution of the universe in the present paper.

The bases of the present model are the general relativity, the conventional quantum field theory for finite temperature and grand unified theory (GUT).

The basic idea of the present model is conjecture 1 in Section 2.

We consider the following condition to be necessary in order to solve the space-time singularity and the cosmological constant problems on the basis of the classical cosmology and the conventional quantum field theory.

Condition: There are two sorts of matter which are symmetric, whose gravitational masses are opposite to each other, although whose masses are all positive.

The two sorts of matter are called solid matter (s-matter) and void matter (v-matter), respectively. The condition implies that if $\rho_{s}=\rho_{v}$, then $\rho_{g s}=-\rho_{g v}$, here $\rho_{g}$ denotes a gravitational mass density. The conditions cannot be realized in the conventional theory, but can be realized in the present model. In order to uniformly solve the above four problems, we present a new conjecture equivalent to the condition and construct two cosmological model, i.e. [19] [20] and this model in the present paper.

The present model has the following results:

1. There is no space-time singularity in this model.

2. It is derived from this model that there are the critical temperature $T_{c r}$, the highest temperature $T_{\max } \geq T_{c r}$, the least scale $R_{\min }>0$ and the largest energy density $\rho_{\max }$ in the universe. Both $V_{0}$ and $T_{c r}$ are new important constants. Both $T_{\max }$ and $\rho_{\max }$ are finite. It is impossible that the Plank temperature $T_{P}$, length $l_{P}$ and time $t_{P}$ are arrived, because $T_{\max } \ll T_{P}$, and $R_{\min }$ is not small. In general, the radius of a local inertial system is so large, $r \gg l_{P}$, that the quantum effects corresponding to $l_{P}$ may be neglected.

3. The evolution of the universe which is derived from this model are consistent with the observations up to now.

There are two sorts of spontaneous symmetry breaking in the present model because of conjecture 1 , and they are called $S$-breaking and $V$-breaking.

According to the present model, the evolving process of space is as follows.

In the S-breaking, space can contract so that temperature $T$ rises. When $T$ arrives the critical temperature $T_{c r}$, the universe is in the most symmetric state with $S U_{S}(5) \times S U_{V}(5)$ symmetry. When space continues to contract so that $T$ arrives the highest temperature $T_{\max }$, space expands and then inflates. After inflation, the most symmetric state transits to the state with the $V$-breaking. After reheating, the evolving process is as follows: Space expands with a deceleration, expands with an acceleration, then expands with a deceleration, finally comes to static and begin to contract, in turn.

4. The relation between the optical distance and the redshift is derived from the present model. It is consistent with the observations up to now.

5. Equations governing nonrelativistic fluid motion are generalized to the present model. Galaxies can form earlier according to this model than that according to the conventional theory.

6. Three predictions are given.

7. Primordial nucleosynthesis and cosmic microwave background radiation are explained.

8. Dark energy is explained as s-matter when the universe is in the $V$-breaking. In contrast with the dark energy, $\rho_{s g}=-\rho_{s}<0$ in the $V$-breaking.

9. $\rho_{\text {gvac }}=\rho_{s, v a c}-\rho_{v, v a c}=0$ is proved, although $\rho_{v a c}=\rho_{s, v a c}+\rho_{v, v a c}$ is still very large. Consequently, $\lambda_{\text {eff }}=\lambda=0$.

Problems 5 and 7 will be discussed in the following paper.

Section 2 is "Conjectures, action, energy-momentum tensor and field equations"; Section 3 is "Spontaneous symmetry breaking"; Section 4 is "Evolution equations"; Section 5 is "Temperature effect"; Section 6 is "Space can contract, but there is no singularity"; Section 7 is "Space inflation"; Section 8 is "Evolving process of space after inflation"; Section 9 is "After expansion with an acceleration, space expands with a deceleration, then comes to static and finally begin to contract". Section 10 is "Existing and distribution forms of $S U_{S}(5)$ color singlets". Section 11 is "New predictions, an inference, and there is no restriction for $T^{\mu \nu}$ "; Section 12 is "Conclusions". 


\section{Conjectures, Action, Energy-Momentum Tensor and Field Equations}

\subsection{Conjectures}

In order to solve the problems mentioned before, we propose the following conjectures:

Conjecture 1 There are two sorts of matter which are called solid-matter (s-matter) and void-matter (v-matter), respectively. Both are symmetric and the symmetric gauge group is $S U_{S}(5) \times S U_{V}(5)$. Both contributions to the Einstein tensor are opposite each other. There is no other interaction between both except interaction (2.10) of s-Higgs fields and v-Higgs fields.

Conjecture 2 When $S U(5)$ symmetry holds, there is the critical temperature $T_{p l}$, all particles exist in $S U$ (5) color singlets when $T<T_{p l}$.

Because of conjecture 1 , there are two sorts of symmetry breaking which are called S-breaking in which $\left\langle\omega_{s}\right\rangle_{0} \neq 0$ and $\left\langle\omega_{v}\right\rangle_{0}=0$ and $V$-breaking in which $\left\langle\omega_{v}\right\rangle_{0} \neq 0$ and $\left\langle\omega_{s}\right\rangle_{0}=0$, here $\omega$ denotes an arbitrary Higgs field. The meanings of conjecture 1 are as follows. The model and its all inferences are invariant when $S \rightleftarrows V$ and $S \rightleftarrows V$. The multiplet of $S U_{S}(5)$ is the same as that of $S U_{V}(5)$; When temperature $T \geq T_{c r}$, s-particles and v-particles are completely symmetric, here $T_{c r}$ is the critical temperature (see section 5 . B); When temperature $T<T_{c r}, S U_{S}(5)$ or $S U_{V}(5)$ is broken. Let $S U_{S}(5)$ be broken, then $S U_{V}(5)$ still holds.

Conjecture 2 holds obviously. In fact, this conjecture is a direct generalization of $S U(3)$ color singlets.

Another premise of the present model is the conventional $S U(5)$ grand unified theory (GUT). But it is easily seen that the present model does not rely on the special GUT. Provided conjecture 1 and such a coupling as (2.10) are kept, the GUT can be applicable.

The gravitational properties of matter and the mode of symmetry breaking determine the features of spacetime. We consider that there are only two possibilities.

A . The first possibility can be described by the conventional theory. There is only one sort of matter so that the equivalence principle strictly holds. This theory is simple, but there must be essential difficulties. For example, there must be the singularity and cosmological constant issues which cannot be solved in the frame of this theory because of the Hawking theorems etc.

B. The basis of the second possibility is conjecture 1 .

We explain it in detail as follows:

1. It must be emphasized that there is no negative mass or negative probability in the present model at all. Conjecture 1 implies that $m_{s g}=-m_{v g}$ when $m_{s}=m_{v}$. In the S-breaking, $m_{s g}=m_{s} \geq 0$ and $m_{v g}=-m_{v} \leq 0$ because of the reasons $6-7$ below. Here $m_{g}$ denotes a gravitational mass. Consequently, both s-energy and $v$-energy must be positive (see (2.20)-(2.21)).

2. The observation basis of conjecture 1 is that space expands with an acceleration now. One of the two sorts of matter must exist in $S U(5)$ color singlets. The color singlets must loosely distribute in whole space, and can cause space to expand with an acceleration, but cannot be observed as so-called dark energy (see 4 - 6 below).

3. Because of conjecture 1 , there must be two sorts of symmetry breaking.

Because of conjecture 1, s-Higgs fields and v-Higgs fields must be symmetric as well. If the symmetry of $s$-matter and $v$-matter was not broken, both s-matter and $v$-matter will exist in the same form at arbitrary time and place. This implies that the nature is simply duplicate. This is impossible because the nature does like duplicate. Of course, this contradicts experiments and observations as well. Consequently the symmetry must be broken when $T<T_{c r}$. Thus the coupling constant $\Lambda$ etc. in (2.10) must be positive so that there must be the two sorts of breaking.

The existing probability of the S-breaking and the V-breaking must be equal because of conjecture 1 . This equality can be realized by two sorts of modes.

(1) The universe is composed of infinite s-cosmic islands with the S-breaking and $v$-cosmic islands with the V-breaking; This possibility has been discussed [19] [20].

(2) The whole universe is in the same breaking (e.g. the S-breaking). But one sort of breaking can transform to another as space contracts to the least scale $R_{\min }$ (see later). We discuss the case in the present paper. The $R W$ metric is applicable to the case.

4 . There is only the repulsion between s-matter and v-matter. Consequently, any bound state is composed of only the s-particles or only the v-particles, i.e. there is no bound state which is composed of the s-particles and 
the v-particles.

Because of conjecture 1, there is the repulsion between s-matter and v-matter and the repulsion constant is the same as the gravitation constant so that the repulsion is weak as the gravitation. The interaction (2.10) is repulsive as well. After reheating, Higgs particles can get very large masses, hence the interaction (2.10) is weak and may be ignored.

5. s-matter and $v$-matter are no longer symmetric after the symmetry breaking.

In the S-breaking, $S U_{S}(5)$ is finally broken to $S U_{S}(3) \times U_{S}(1)$ and $S U_{V}(5)$ holds all the time. Consequently, s-particles get their masses and form s-atoms, s-observers and s-galaxies etc.; while all $v$-fermions and v-gauge bosons are still massless and must form $S U_{V}(5)$ color-singlets after reheating.

There is no interaction (e.g. the electroweak interaction) except the gravitation among the $S U_{V}(5)$ colorsinglets, because $S U(5)$ is a simple group. Hence the $S U_{V}(5)$ color-singlets cannot form $v$-atoms and $v$ galaxies etc., and must distribute loosely in space as the so-called dark energy.

Thus, in the S-breaking, s-matter is identified with the conventional matter, while $v$-matter is similar to dark energy. In contrast with the dark energy, the gravitational masses of $v$-matter is negative.

6. The $S U_{V}(5)$ color-single states cannot be observed by an s-observer.

As mentioned above, there is only the repulsion between s-matter and v-matter The repulsions originating from conjecture 1 and (2.10) are very weak after reheating. The v-particles can only form the $S U_{V}(5)$ color singlets with their very small masses. The $S U_{V}(5)$ color singlets cannot form atoms and galaxies etc., and can only distribute loosely in space. On the other hand, $\rho_{v}$ must be very small when $\rho_{s}$ is very large because of the repulsion. Consequently, in fact, it is impossible to observe the $S U_{V}(5)$ color-singlets even by the repulsion as well.

In the S-breaking, only the cosmological effects of v-matter are important and are consistent with the observation up to now.

7. The equivalence principle still strictly holds for the s-particles $\left(m_{s g}=m_{s}\right)$, but is violated by the $v$-particles $\left(m_{v g}=-m_{v}\right)$ in the S-breaking. But the motion equations of all s-particles and all v-particles are still independent of their masses.

In the S-breaking, there are only s-observers and s-galaxies, and there is no v-observer and v-galaxy. Hence the gravitational masses of s-particles must be positive, i.e. $m_{s g}=m_{s}>0$, while the gravitational masses of $v$-matter must be negative relatively to s-matter, i.e. $m_{v g}=-m_{v}<0$, because of conjecture 1 . Thus, a s-photon falling in a gravitational field must have 'purple shift', but a $v$-particle (there is no $v$-photon and there are only the $S U_{V}(5)$ color singlets) falling in the same gravitational field will have 'redshift'.

Although the equivalence principle is violated by $v$-particles in the $S$-breaking, there is no contradiction with any observation and experiment, because the $S U_{V}(5)$ color singlets cannot be observed by a s-observer (see 6).

8. When temperature is high enough, the expectation values of Higgs fields are small so that all masses of Higgs particles are small. Thus, $\rho_{s}$ and $\rho_{v}$ can transform from one into another by (2.10). Consequently, space cannot contract to infinite small and inflation must occur.

The interaction (2.10) can be neglected after reheating, because the masses of the Higgs particles are very large in low temperatures. Thus, the transformation of s-particles and v-particles from one into another may be neglected.

In summary, in the S-breaking, the $S U_{V}(5)$ color singlets cannot be observed and have only the cosmological effects. Conjecture 1 does not contradict any experiment and observation up to now.

We will see in the following that the evolution of the universe can be well explained, and the singularity and cosmological constant issues can be solved.

\subsection{Action}

The breaking mode of the symmetry is only one of the S-breaking and the V-breaking due to (2.10). In the $S$-breaking, there are only s-observators. Analogously, in the V-breaking, there are only v-observators. Hence the actions should be written as two sorts of form, $I_{S}$ in the S-breaking and $I_{V}$ in the $V$-breaking. Of course, only one of both $I_{S}$ and $I_{V}$ can describe the evolution of the universe. Hence, in any case, the action is unique. But the S-breaking can transform to the $V$-breaking when temperature is high enough, hence both $I_{S}$ and $I_{V}$ are necessary. Because of conjecture 1 , the structures of $I_{S}$ and $I_{V}$ are the same, i.e. $I_{S} \rightleftarrows I_{V}$ when $S \rightleftarrows V$ and $S \rightleftarrows V$. Thus, at the zero-temperature, we have 


$$
\begin{aligned}
& I_{S}=I_{g}+I_{S M}=I_{g}+I_{V M}=I_{V}, \\
& I_{g}=\frac{1}{16 \pi G}\left(\int_{\Sigma} R \sqrt{-g} \mathrm{~d}^{4} x+2 \int_{\partial \Sigma} K \sqrt{ \pm h} \mathrm{~d}^{3} x\right), \\
& I_{V M}=\int \mathrm{d}^{4} x \sqrt{-g} \mathcal{L}_{V M}, \mathcal{L}_{V M}=\alpha\left(\mathcal{L}_{v}+V_{0}\right)+\beta \mathcal{L}_{s}+\frac{1}{2}(\alpha+\beta) V_{v s}, \\
& I_{V M}=\int \mathrm{d}^{4} x \sqrt{-g} \mathcal{L}_{V M}, \mathcal{L}_{V M}=\alpha\left(\mathcal{L}_{v}+V_{0}\right)+\beta \mathcal{L}_{s}+\frac{1}{2}(\alpha+\beta) V_{v s}, \\
& \mathcal{L}_{s}=\mathcal{L}_{s M}\left(\Psi_{s}, g(x), g(x),{ }_{\mu}\right)+V_{s}\left(\omega_{s}\right), \\
& \mathcal{L}_{v}=\mathcal{L}_{v M}\left(\Psi_{v}, g(x), g(x),{ }_{\mu}\right)+V_{v}\left(\omega_{v}\right), \\
& V_{s v}\left(\omega_{s}, \omega_{v}\right)=V_{v s}\left(\omega_{s}, \omega_{v}\right) ; \\
& \omega_{s} \equiv \Omega_{s}, \Phi_{s}, \chi_{s} ; \omega_{v} \equiv \Omega_{v}, \Phi_{v}, \chi_{v},
\end{aligned}
$$

where the meanings of the symbols are as follows: $g=\operatorname{det}\left(g_{\mu v}\right)$, and $g_{\mu v}=\operatorname{diag}(-1,1,1,1)$ in flat space. $R$ is the scalar curvature. Here $\alpha$ and $\beta$ are two parameters, may be called "gravitation charges", and are finally taken as $\alpha=-\beta=1 . V_{0}$ in $\mathcal{L}_{S M}\left(\mathcal{L}_{V M}\right)$ is a parameter in order to determine the zero-point of the potential $V_{s}\left(V_{v}\right) . \mathcal{L}_{s M}\left(\mathcal{L}_{v M}\right)$ is the Lagrangian density of all s-fields ( $v$-fields) and their couplings of the $S U$ (5) GUT except the Higgs potentials $V_{s}, V_{v}$ and $V_{s v} . \Psi_{s}$ and $\Psi_{v}$ represents all s-fields and all $v$-fields, respectively. For a boson field, $\Psi$ denotes its covariant derivative as well. Both $\mathcal{L}_{s}$ and $\mathcal{L}_{s}$ do not contain the contribution of the gravitation energy and the repulsion energy. It may be seen that the set of equation (2.1)-(2.7) is unchanged when the subscripts $s \rightleftarrows V$ and $S \rightleftarrows V$. This shows the symmetry of $s$-matter and $v$-matter. The physics quantities with the subscript ' $S$ ' (' $V$ ') denotes that they have meaning only when the universe is in the $S(' V$ ')-breaking. It is the same for the subscript ' $V$ ' as for ' $S$ '. For simplicity, the subscripts ' $S$ ' and ' $V$ ' may be elided in the following when there is no confusion.

Gibbons and Hawking pointed out that in order to get the Einstein field equations [21], it is necessary that

$$
I_{g}^{\prime}=\frac{1}{16 \pi G} \int_{\Sigma} R \sqrt{-g} \mathrm{~d}^{4} x \rightarrow I_{g}=\frac{1}{16 \pi G}\left(\int_{\Sigma} R \sqrt{-g} \mathrm{~d}^{4} x+2 \int_{\partial \Sigma} K \sqrt{ \pm h} \mathrm{~d}^{3} x\right) .
$$

This is because it is not necessary that $\delta \Gamma_{\mu \nu}^{\alpha}=0$ on the boundary $\partial \Sigma$. Hence $I_{g}^{\prime}$ is replaced by $I_{g}$ in (2.2). $\Sigma$ is a manifold with four dimensions. $\partial \Sigma$ is the boundary of $\Sigma . \quad K=t r K_{j}^{i} . \quad K_{i j}=-\nabla_{i} n_{j}$ is the outer curvature on $\partial \Sigma$. $n_{j}$ is the vertical vector on $\partial \Sigma$. $h=\left|h_{i j}\right|$, and $h_{i j}$ is the induced outer metric on $\partial \Sigma$. When $\partial \Sigma$ is space-like, $\sqrt{ \pm h}$ takes positive sign. When $\partial \Sigma$ is time-like, $\sqrt{ \pm h}$ takes negative sign.

The Higgs potentials in (2.5)-(2.7) is the following:

$$
\begin{aligned}
V_{s}= & -\frac{1}{2} \mu^{2} \Omega_{s}^{2}+\frac{1}{4} \lambda \Omega_{s}^{4}-\frac{1}{2} w \Omega_{s}^{2} \operatorname{Tr} \Phi_{s}^{2}+\frac{1}{4} a\left(\operatorname{Tr} \Phi_{s}^{2}\right)^{2} \\
& +\frac{1}{2} b \operatorname{Tr}\left(\Phi_{s}^{4}\right)-\frac{1}{2} \varsigma \Omega_{s}^{2} \chi_{s}^{+} \chi_{s}+\frac{1}{4} \xi\left(\chi_{s}^{+} \chi_{s}\right)^{2}, \\
V_{v}= & -\frac{1}{2} \mu^{2} \Omega_{v}^{2}+\frac{1}{4} \lambda \Omega_{v}^{4}-\frac{1}{2} w \Omega_{v}^{2} \operatorname{Tr} \Phi_{v}^{2}+\frac{1}{4} a\left(\operatorname{Tr} \Phi_{v}^{2}\right)^{2} \\
& +\frac{1}{2} b \operatorname{Tr}\left(\Phi_{v}^{4}\right)-\frac{1}{2} \varsigma \Omega_{v}^{2} \chi_{v}^{+} \chi_{v}+\frac{1}{4} \xi\left(\chi_{v}^{+} \chi_{v}\right)^{2}, \\
V_{s v}= & \frac{1}{2} \Lambda \Omega_{s}^{2} \Omega_{v}^{2}+\frac{1}{2} p \Omega_{s}^{2} \operatorname{Tr} \Phi_{v}^{2}+\frac{1}{2} q \Omega_{s}^{2} \chi_{v}^{+} \chi_{v}+\frac{1}{2} p \Omega_{v}^{2} \operatorname{Tr} \Phi_{s}^{2}+\frac{1}{2} q \Omega_{v}^{2} \chi_{s}^{+} \chi_{s},
\end{aligned}
$$

where $\Omega_{a}, \Phi_{a}=\sum_{i=1}^{24}\left(T_{i} / \sqrt{2}\right) \varphi_{a i}$ and $\chi_{a}$ are respectively $\underline{1}, \underline{24}$ and $\underline{5}$ dimensional representations of the $S U(5)$ group, $T_{i}^{\prime} s$ are the $S U(5)$ generators, $a=s, v$. Here the couplings of $\Phi_{a}$ and $\chi_{a}$ are ignored for short [22] [23]. (2.8) is the same as that in [22] [23]. The coupling constants in (2.8)-(2.10) are all positive, especially, as mentioned before, $\Lambda, p$ and $q$ in (2.10) must be positive.

We do not consider the terms coupling to curvature scalar, e.g. $\xi R \Omega^{2}$, for a time. In fact, $\xi R\left(\Omega_{s}^{2}-\Omega_{v}^{2}\right) \sim 0$ when temperature $T$ is high enough due to the symmetry between s-matter and $v$-matter. 


\subsection{Energy-Momentum Tensors and Field Equations}

By the conventional method, from (2.2) we can get

$$
\delta I_{g}=\frac{1}{16 \pi G} \int_{\Sigma}\left(R_{\mu v}-\frac{1}{2} g_{\mu v} R\right) \delta g^{\mu v} \sqrt{-g} \mathrm{~d}^{4} x .
$$

Considering $\alpha=-\beta=1$, from (2.3)-(2.4) we have

$$
\begin{aligned}
\delta I_{S M} & =\int \frac{1}{\sqrt{-g}}\left[\frac{\partial \mathcal{L}_{S M} \sqrt{-g}}{\partial g^{\mu \nu}}-\left(\frac{\partial \mathcal{L}_{S M} \sqrt{-g}}{\partial g_{, \sigma}^{\mu \nu}}\right)_{, \sigma}\right] \delta g^{\mu \nu} \sqrt{-g} \mathrm{~d}^{4} x \\
& =\int \frac{1}{2}\left[T_{s \mu \nu}-g_{\mu \nu} V_{0}-T_{v \mu \nu}\right] \delta g^{\mu \nu} \sqrt{-g} \mathrm{~d}^{4} x, \\
\delta I_{V M} & =\int \frac{1}{2}\left[T_{v \mu \nu}-g_{\mu \nu} V_{0}-T_{s \mu \nu}\right] \delta g^{\mu \nu} \sqrt{-g} \mathrm{~d}^{4} x, \\
T_{a \mu \nu}= & T_{a M \mu \nu}-g_{\mu \nu} V_{a}, a=s \text { or } v, \\
T_{a M \mu \nu}= & \frac{2}{\sqrt{-g}}\left[\frac{\partial\left(\sqrt{-g} \mathcal{L}_{a M}\right)}{\partial g^{\mu \nu}}-\left(\frac{\partial\left(\sqrt{-g} \mathcal{L}_{a M}\right)}{\partial g_{, \sigma}^{\mu \nu}}\right)_{, \sigma}\right] .
\end{aligned}
$$

From (2.11)-(2.13) we obtain

$$
R_{\mu v}-\frac{1}{2} g_{\mu v} R=-8 \pi G T_{A g \mu v}, A=S \text { or } V
$$

In the S-breaking,

$$
\begin{aligned}
& T_{S g \mu v} \equiv T_{s \mu \nu}-g_{\mu \nu} V_{0}-T_{v \mu \nu}=T_{S M g \mu \nu}-g_{\mu \nu} V_{S g} \\
& T_{S M g \mu v} \equiv T_{S M \mu \nu}-T_{v M \mu \nu}, V_{S g}=V_{s}+V_{0}-V_{v} .
\end{aligned}
$$

In the V-breaking,

$$
\begin{aligned}
& T_{V g \mu \nu} \equiv T_{v \mu \nu}-g_{\mu \nu} V_{0}-T_{s \mu \nu}=T_{V M g \mu \nu}-g_{\mu \nu} V_{V g} \\
& T_{V M g \mu v} \equiv T_{v M \mu \nu}-T_{s M \mu \nu}, V_{V g}=V_{v}+V_{0}-V_{s} .
\end{aligned}
$$

$T_{A g \mu v}, \quad T_{A M g \nu}$ and $V_{A g}$ are the gravitational energy-momentum tensor density, the gravitational energymomentum tensor density without the Higgs potential and the gravitational potential density of the Higgs fields in the A-breaking, respectively.

It is seen from (2.17)-(2.18) that $V_{A g}$ is independent of $V_{s v}$. This implies that the potential energy $V_{s v}$ is different from other energies in essence. There is no contribution of $V_{s v}$ to $R_{\mu v}$, i.e., there is no gravitation and repulsion of the potential energy $V_{s v}$. This does not satisfy the equivalence principle. But this does not cause any contradiction with all given experiments and astronomical observations, because $V_{s v}=0$ in either of breaking modes.

We will see that, in fact, $V_{v \min }\left(\varpi_{v}\right)=0$ because $\left\langle\omega_{v}\right\rangle=0$ in the $S$-breaking, and $V_{s \min }\left(\varpi_{s}\right)=0$ because $\left\langle\omega_{s}\right\rangle=0$ in the $V$-breaking. Hence

$$
V_{A g}=V_{a \min }\left(\varpi_{s}\right)+V_{0}=V_{0} \text { when } \varpi_{s}=\varpi_{v}=0 \text {. }
$$

From (2.1) the energy-momentum tensor density which does not contain the energy-momentum tensor of gravitational and repulsive interactions can be defined as

$$
\begin{aligned}
T_{A \mu v} & =\frac{2}{\sqrt{-g}}\left(\frac{\partial}{\partial \alpha}+\frac{\partial}{\partial \beta}\right) \cdot\left[\frac{\partial\left(\sqrt{-g} \mathcal{L}_{A}\right)}{\partial g^{\mu \nu}}-\left(\frac{\partial\left(\sqrt{-g} \mathcal{L}_{A}\right)}{\partial g_{, \sigma}^{\mu v}}\right)_{, \sigma}\right] \\
& \equiv T_{s \mu \nu}+T_{v \mu \nu}-g_{\mu \nu}\left(V_{s v}+V_{0}\right)=T_{A M \mu \nu}-g_{\mu \nu} V_{A} \equiv T_{\mu v},
\end{aligned}
$$




$$
\begin{aligned}
& T_{A M \mu \nu}=T_{S M \mu \nu}+T_{v M \mu \nu} \equiv T_{M \mu \nu}, \\
& V_{A}=V_{s}+V_{v}+V_{s v}+V_{0} \equiv V .
\end{aligned}
$$

As mentioned before, $\alpha$ and $\beta$ in (2.3)-(2.4) may be regarded as the gravitation charges. The the gravitation charges of $T_{s \mu \nu}, T_{v \mu v}$ and $g_{\mu \nu} V_{s v}$ are regarded as $1,-1$ and 0 in the $S$-breaking, respectively. The energy-momentum tensor should be independent of the gravitation charges, because the energy-momentum tensor of the gravitation fields is not considered. Hence it is necessary to eliminate $\alpha$ and $\beta$ from the definition of $T_{\mu \nu}$ by the operator $(\partial / \partial \alpha+\partial / \partial \beta)$. The operator is the only difference between the definition of $T_{\mu v}$ in this model and that in the conventional theory. This definition does not contradict any basic principle and it is completely consistent with the conventional theory. In fact, there is one sort of matter in the conventional theory (i.e. $\left.T_{v \mu \nu}=g_{\mu \nu} V_{s v}=0\right)$ so that $(\partial / \partial \alpha+\partial / \partial \beta)$ can be reduced to $(\partial / \partial \alpha)\left(\alpha T_{s \mu \nu}\right)=T_{s \mu \nu}=T_{\mu \nu}$.

It is seen from (2.20)-(2.21) that both s-energy and v-energy must be positive.

It should be pointed out that only (2.16) and (2.17) is applicable in the S-breaking, and only (2.16) and (2.18) applicable in the $V$-breaking.

Considering $I_{S M}\left(I_{V M}\right)$ to be a scalar [24] or considering

$$
\left(R^{\mu v}-\frac{1}{2} g^{\mu v} R\right)_{; \nu}=\left(g^{\mu v} V_{0}\right)_{; \nu}=0
$$

and (2.16) and (2.14) we obtain

$$
T_{S g ; \nu}^{\mu \nu}=T_{s ; \nu}^{\mu \nu}-T_{v ; v}^{\mu \nu}=-T_{V g ; \nu}^{\mu \nu}=0
$$

\subsection{The Difference of Motion Equations of a $v$-Particle and a $s$-Particle in the Same Gravitational Field}

From (2.16) we have

$$
\left(R^{\mu \nu}-\frac{1}{2} g^{\mu \nu} R\right)_{; \nu}=0=T_{A g ; v}^{\mu v}=\frac{1}{\sqrt{-g}} \frac{\partial}{\partial x^{v}}\left(\sqrt{-g} T_{A g}^{\mu \nu}\right)+\Gamma_{\alpha \nu}^{\mu} T_{A g}^{\alpha \nu} .
$$

In the S-breaking, $T_{A g}^{\mu \nu}=T_{S g}^{\mu \nu}=T_{s g}^{\mu \nu}+T_{v g}^{\mu \nu}, T_{s g}^{\mu \nu}=T_{s}^{\mu \nu}$ and $T_{v g}^{\mu \nu}=-T_{v}^{\mu \nu}$.

Consider a point-particle with its gravitational mass $m_{g}$ to move in a gravitational field with its strength $\Gamma_{\alpha \nu}^{\mu}$. From (2.23) we can get

$$
m_{g} \frac{\mathrm{d}^{2} x^{\mu}}{\mathrm{d} \tau^{2}}+m_{g} \Gamma_{\alpha \beta}^{\mu} \frac{\mathrm{d} x^{\alpha}}{\mathrm{d} \tau} \frac{\mathrm{d} x^{\beta}}{\mathrm{d} \tau}=0=\frac{\mathrm{d}^{2} x^{\mu}}{\mathrm{d} \tau^{2}}+\Gamma_{\alpha \beta}^{\mu} \frac{\mathrm{d} x^{\alpha}}{\mathrm{d} \tau} \frac{\mathrm{d} x^{\beta}}{\mathrm{d} \tau}, \quad m_{g}=m_{s g}, m_{v g}
$$

It is seen from (2.24) that the motion equation of the gravitation mass $m_{v g}$ is the same as that of the gravitation mass $m_{s g}$. This is the same as the conventional theory.

It must be given one's attention to that (2.24) is only the equation of a gravitation mass $m_{g}$, but is not the equation of an inertial mass $m_{I}$. According to the equvalence principle in the conventional theory, $m_{I}=m_{g}$, here $m_{I}$ is the inertial mass. Consequently, (2.24) is the equation of an inertial mass $m_{I}$ as well.

According to the present model, because of conjecture 1, the gravitational field equation can determine only the motion equation of a gravitation mass (2.24), but cannot determine the motion equation of an inertial mass $m_{I}$. The motion equation of an inertial mass $m_{I}$ must be determined on the bases of conjecture 1 and the gravitational field equation.

In the S-breaking, according to conjecture $1, m_{s I} \equiv m_{s}=m_{s g}$ and $m_{v I} \equiv m_{v}=-m_{v g}$. Hence the equation of $m_{s}$ is the same as that of $m_{s g}$, i.e. (2.24), but the equation of $m_{v I}$ must be different from that of $m_{v g}$. According to conjecture 1, both $m_{s I}$ and $m_{v I}$ are positive, and $m_{s g}=-m_{v g}$ when $m_{s}=m_{v} \cdot m_{g} \Gamma_{\alpha \beta}^{\mu}$ in (2.24) is the coupling of the gravitational charge $m_{g}$ and the gravitational field with its strength $\Gamma_{\alpha \nu}^{\mu}$. The second term in (2.24) determines the force acting on $m_{v I}$. Considering $m_{v I}=-m_{v g}$ in the S-breaking so that the acceleration of $m_{v I}$ is opposite to that of $m_{v g}$, we get the motion equation of $m_{v I}$ in the gravitational field with its strength $\Gamma_{\alpha v}^{\mu}$ to be

$$
\left(-m_{v g}\right) \frac{\mathrm{d}^{2} x^{\mu}}{\mathrm{d} \tau^{2}}+m_{v g} \Gamma_{\alpha \beta}^{\mu} \frac{\mathrm{d} x^{\alpha}}{\mathrm{d} \tau} \frac{\mathrm{d} x^{\beta}}{\mathrm{d} \tau}=0=-\frac{\mathrm{d}^{2} x^{\mu}}{\mathrm{d} \tau^{2}}+\Gamma_{\alpha \beta}^{\mu} \frac{\mathrm{d} x^{\alpha}}{\mathrm{d} \tau} \frac{\mathrm{d} x^{\beta}}{\mathrm{d} \tau} .
$$


Comparing (2.24) and (2.25), we see that in the same gravitational field, the motion equation of a s-particle is different from that of a $v$-particle.

Analogous to the case in the $S$-breaking, in the V-breaking, because of the symmetry of s-matter and $v$-matter, we have

$$
\begin{gathered}
\frac{\mathrm{d}^{2} x^{\mu}}{\mathrm{d} \tau^{2}}+\Gamma_{\alpha \beta}^{\mu} \frac{\mathrm{d} x^{\alpha}}{\mathrm{d} \tau} \frac{\mathrm{d} x^{\beta}}{\mathrm{d} \tau}=0, \text { for a v-particle, } \\
-\frac{\mathrm{d}^{2} x^{\mu}}{\mathrm{d} \tau^{2}}+\Gamma_{\alpha \beta}^{\mu} \frac{\mathrm{d} x^{\alpha}}{\mathrm{d} \tau} \frac{\mathrm{d} x^{\beta}}{\mathrm{d} \tau}=0, \text { for a s-particle. }
\end{gathered}
$$

Considering the Newtonian approximation, i.e. the velocity of a particle is low $(v / c \ll 1)$, a gravitational field is weak $\left(g_{\mu v}=\eta_{\mu v}+h_{\mu v}, \quad\left|h_{\mu v}\right| \ll 1\right)$ and static $\left(g_{\mu v, 0}=0\right)$, and $\left|h_{\mu v, i}\right| \ll 1$, from (2.24) we have

$$
\begin{aligned}
& \frac{\mathrm{d}}{\mathrm{d} \tau}\left(m_{g} \frac{\mathrm{d} x^{0}}{\mathrm{~d} \tau}\right)=0, \\
& \frac{\mathrm{d}}{\mathrm{d} \tau}\left(m_{g} \frac{\mathrm{d} x^{i}}{\mathrm{~d} \tau}\right)+m_{g} \Gamma_{00}^{i}\left(\frac{\mathrm{d} x^{0}}{\mathrm{~d} \tau}\right)^{2}=0 .
\end{aligned}
$$

From (2.28), $x^{0}=a \tau+b, \quad a$ and $b$ are two constants. Considering $\Gamma_{\alpha \beta}^{\mu}=\left(\eta^{\mu \sigma} / 2\right)\left(h_{\sigma \alpha, \beta}+h_{\sigma \beta, \alpha}-h_{\alpha \beta, \sigma}\right)$, and let $\varphi=-h_{00} / 2$ be Newtonian gravitational potential, we can reduce (2.29) to

$$
m_{g} \frac{\mathrm{d}^{2} x^{i}}{\mathrm{~d} t^{2}}=\frac{m_{g}}{2} h_{00}=m_{g}\left(-\frac{\partial \varphi}{\partial x^{i}}\right), x^{0}=t .
$$

Let $\varphi$ is caused by a static and spheral-symmetric s-object with its mass $M$. In the S-breaking, $\varphi=-G M / r$. Thus, from (2.24)-(2.25) and (2.30) we get

$$
\begin{aligned}
\frac{\mathrm{d}^{2} x^{i}}{\mathrm{~d} t^{2}} & =G \frac{\partial}{\partial x^{i}}(M / r) \text { for a s-particle, } \\
\frac{\mathrm{d}^{2} x^{i}}{\mathrm{~d} t^{2}} & =-G \frac{\partial}{\partial x^{i}}(M / r) \text { for a v-particle. }
\end{aligned}
$$

Let $\varphi$ is caused by a static and spheral-symmetric v-object with its mass $M$. In the S-breaking, $\varphi=G M / r$ because conjecture 1. Thus, from (2.24)-(2.25) and (2.30) we get

$$
\begin{aligned}
\frac{\mathrm{d}^{2} x^{i}}{\mathrm{~d} t^{2}} & =-G \frac{\partial}{\partial x^{i}}(M / r) \text { for a s-particle, } \\
\frac{\mathrm{d}^{2} x^{i}}{\mathrm{~d} t^{2}} & =G \frac{\partial}{\partial x^{i}}(M / r) \text { for a } v \text {-particle. }
\end{aligned}
$$

It is seen that the motion equation of a v-particle in such a gravitational field caused by $v$-matter is the same as that of a s-particle in the gravitational field caused by s-matter in the Newtonian approximation, when the distributing mode of $v$-matter is the same as that of s-matter.

In the $V$-breaking, we can get the same results as above, provided $S \rightleftarrows V$ and $s \rightleftarrows V$.

\section{Spontaneous Symmetry Breaking}

Ignoring the couplings of $\Phi_{s}$ and $\chi_{s}$ and suitably choosing the parameters of the Higgs potential, analogously to Ref. [22] [23], we can prove from (2.8)-(2.10) that there are the following vacuum expectation values (the $S$-breaking) at the zero-temperature and under the tree-level approximation

$$
\begin{aligned}
& \left\langle 0\left|\omega_{v}\right| 0\right\rangle \equiv \bar{\omega}_{v 0}=0,\left\langle 0\left|\omega_{s}\right| 0\right\rangle \equiv \bar{\omega}_{s 0} \neq 0, \\
& \left\langle 0\left|\Omega_{s}\right| 0\right\rangle=v_{\Omega 0}, \\
& \left\langle 0\left|\Phi_{s}\right| 0\right\rangle=\operatorname{Diagonal}\left(1,1,1,-\frac{3}{2},-\frac{3}{2}\right) v_{\varphi 0},
\end{aligned}
$$




$$
\left\langle 0\left|\chi_{s}\right| 0\right\rangle^{+}=\frac{v_{\chi 0}}{\sqrt{2}}(0,0,0,0,1),
$$

Ignoring the contributions of $\Phi_{s}$ and $\chi_{s}$ to $\left\langle 0\left|\Omega_{s}\right| 0\right\rangle$, at the zero-temperature we get

$$
\begin{aligned}
v_{\Omega 0}^{2} & =\frac{\mu^{2}}{f}, \quad f \equiv \lambda-\frac{15 w^{2}}{(15 a+7 b)}-\frac{\varsigma^{2}}{\xi} . \\
v_{\varphi 0}^{2} & =\frac{2 w}{15 a+7 b} v_{\Omega 0}^{2}, \\
v_{\chi 0}^{2} & =\frac{2 \zeta}{\xi} v_{\Omega 0}^{2} .
\end{aligned}
$$

We take $\Lambda>\lambda>15 w^{2} /(15 a+7 b)+\zeta^{2} / \xi$. From (2.9)-(2.10) and (3.1)-(3.7) it can be proved that all $v$-Higgs bosons can get their big enough masses. The masses of the Higgs particles exclusive of the $\Phi_{s}$-particles and the $\chi_{s}$-particles in the $S$-breaking are respectively

$$
\begin{aligned}
& m^{2}\left(\Omega_{s}\right)=2 \mu^{2}, \\
& m^{2}\left(\Omega_{v}\right)=\Lambda v_{\Omega 0}^{2}-\mu^{2}, \\
& m^{2}\left(\Phi_{v}\right)=\frac{1}{2} p v_{\Omega 0}^{2}, \\
& m^{2}\left(\chi_{v}\right)=q v_{\Omega 0}^{2} .
\end{aligned}
$$

We can choose such parameters that

$$
m\left(\Omega_{s}\right) \simeq m\left(\Omega_{v}\right) \gg m\left(\varphi_{v}\right) \sim m \varphi\left(\phi_{s}\right) \gg m\left(\chi_{v}\right) \sim m\left(\chi_{s}\right),
$$

e.g., $m\left(\Omega_{s}\right) \sim 10^{16} \mathrm{Gev}, \quad m\left(\varphi_{s}\right) \sim 10^{14} \mathrm{Gev}$ and $m\left(\chi_{s}\right) \sim 10^{2} \mathrm{Gev}$. It is easily seen from (3.8)-(3.11) that all real components of $\Phi_{v}$ have the same mass $m\left(\Phi_{v}\right)$, and all real components of $\chi_{v}$ have the same mass $m\left(\chi_{v}\right)$ in the S-breaking.

The $S$-breaking and the $V$-breaking are symmetric because s-matter and $v$-matter are symmetric. Hence when $s \rightleftarrows V$ and $S \rightleftarrows V$ in (3.1)-(3.12), the formulas are still kept.

Let

we have

$$
V_{s}(0)-V_{s}\left(\varpi_{s 0}\right) \equiv V_{0}-\left(-V_{1}\right) \geq V_{0},
$$

$$
\begin{aligned}
& V_{s}\left(\varpi_{s 0}\right)=-\left(V_{0}+V_{1}\right)=V_{v}\left(\varpi_{v 0}\right), \\
& V_{S g}\left(\varpi_{s 0}, 0\right)=V_{s}\left(\varpi_{s 0}\right)+V_{0}=-V_{1}=V_{V g}\left(0, \varpi_{v 0}\right) .
\end{aligned}
$$

It is easily seen that $V_{0}+V_{1}$ is strictly determined by $V_{s}\left(\varpi_{s}\right)$, but $V_{0}$ or $V_{1}$ is a undetermined parameter. $\left(-V_{1}\right)$ is the zero point of $V_{s}\left(\varpi_{s}\right)$ and $V_{v}\left(\varpi_{v}\right)$. We take $V_{1}$ to be so small that it may be neglected when $\rho_{a}>0$ in the A-breaking, $a=s, v$ and $A=S, V$.

\section{Evolution Equations}

\subsection{Evolution Equations of $R$ in RW Metric}

As is well known, based on the RW metric metric,

$$
(\mathrm{d} s)^{2}=-(\mathrm{d} t)^{2}+R^{2}(t)\left\{\frac{(\mathrm{d} r)^{2}}{1-k r^{2}}+(r \mathrm{~d} \theta)^{2}+(r \sin \theta \mathrm{d} \varphi)^{2}\right\} .
$$

In the present model, we take $k=-1$. Taking $k=0$ or 1 , we can get the results similar to those when $k=-1$. We will discuss the two cases in the following paper. In fact, it is possible that $k$ is changeable with the gravitational mass density $\rho_{g}$. In this case, the results of the present model are more easily obtained [19] [20]. 
Matter in the universe may approximately be regarded as ideal gas distributed evenly in space. Considering the potential energy densities in (2.14), we can write $T_{a \mu v}$ as

$$
\begin{aligned}
& T_{a \mu \nu}=\left[\tilde{\rho}_{a}+\tilde{p}_{a}\right] U_{a \mu} U_{a v}+\tilde{p}_{a} g_{\mu \nu}, \\
& \tilde{\rho}_{a}=\rho_{a}+V_{a}\left(\varpi_{a}\right), \quad \tilde{p}_{a}=p_{a}-V_{a}\left(\varpi_{a}\right),
\end{aligned}
$$

where $U_{a \mu}$ is a 4-velocity and $a=s$ or $v$. In comoving coordinates $U_{a \mu}=\delta_{\mu}^{0}=U_{\mu}$ in a comoving coordinates. $\left(-g_{\mu v} V_{0}\right)$ can be written as

$$
-g_{\mu \nu} V_{0}=\left(\tilde{\rho}\left(V_{0}\right)+\tilde{p}\left(V_{0}\right)\right) U_{\mu} U_{v}+g_{\mu \nu} \tilde{p}\left(V_{0}\right), \tilde{\rho}\left(V_{0}\right)=V_{0}, \tilde{p}\left(V_{0}\right)=-V_{0} .
$$

Considering $U_{\mu}=\delta_{\mu}^{0}$, substituting (4.2)-(4.4) and the RW metric in (4.1) into (2.16), we get the evolution equations

$$
\begin{aligned}
& \dot{R}^{2}+k=\eta\left[\rho_{g}+V_{g}\right] R^{2}, \eta \equiv 8 \pi G / 3, \\
& \ddot{R}=-\frac{1}{2} \eta\left[\left(\rho_{g}+3 p_{g}\right)-2 V_{g}\right] R .
\end{aligned}
$$

In the S-breaking,

$$
\rho_{g}=\rho_{s}-\rho_{v}, p_{g}=p_{s}-p_{v}, V_{g}=V_{s}+V_{0}-V_{v} .
$$

In the V-breaking,

$$
\rho_{g}=\rho_{v}-\rho_{s}, p_{g}=p_{v}-p_{s}, V_{g}=V_{v}+V_{0}-V_{s} .
$$

Comparing (4.5)-(4.6) with the Friedmann equations, we see that provided $\rho, p$ and $V$ in the Friedmann equations are replaced by $\rho_{g}, p_{g}$ and $V_{g},(4.5)-(4.6)$ are obtained.

\subsection{Evolution Equation of $\rho_{g}$}

In contrast with the conventional theory, it is possible that $\dot{\rho} \neq 0$ although $\dot{R}=\dot{V}_{g}=0$. This is because $\rho_{s}$ and $\rho_{v}$ can transform from one to another by (2.10), especially when temperature is high enough (see section $6 B)$.

Let $\rho_{s v}=\rho_{v s}$, e.g. $\rho_{s v}=\rho_{v s}=V_{s v} / 2$, it is obvious that in the $S$-breaking,

$$
\rho_{g}=\rho_{s}-\rho_{v}=\left(\rho_{s}+\rho_{s v}\right)-\left(\rho_{v}+\rho_{v s}\right) .
$$

When $\dot{R}=\dot{V}_{g}=0, \quad \rho=\rho_{s}+\rho_{v}+2 \rho_{v s}$ is the total energy density and is conservational, i.e.

$$
\left(\rho_{s}(t)+\rho_{s v}(t)\right)+\left(\rho_{v}(t)+\rho_{v s}(t)\right)=\left(\rho_{s}\left(t_{0}\right)+\rho_{s v}\left(t_{0}\right)\right)+\left(\rho_{v}\left(t_{0}\right)+\rho_{v s}\left(t_{0}\right)\right) .
$$

It is possible that $\dot{\rho}_{s v} \neq \dot{\rho}_{v s}$ although $\rho_{s v}=\rho_{v s}$. This is because in general, $T_{s} \neq T_{v}$ and $m_{s j} \neq m_{v j}$, here $m_{s j}\left(m_{v j}\right)$ is the mass of a sort of s-particles (v-particles). If $\Delta\left(\rho_{s}+\rho_{s v}\right)$ transforms into the energy density of $v$-particles and $\Delta\left(\rho_{v}+\rho_{v s}\right)$ transforms into the energy density of s-particles in an interval of time $\Delta t$, there must be

$$
\left(\Delta \rho_{g}\right)_{t r}=2\left[\Delta\left(\rho_{s}+\rho_{s v}\right)-\Delta\left(\rho_{v}+\rho_{v s}\right)\right]_{t r} .
$$

Consequently, we have

$$
\dot{\rho}_{g, t r} \equiv\left(\frac{\mathrm{d} \rho_{g}}{\mathrm{~d} t}\right)_{t r}=2\left[\frac{\mathrm{d}\left(\rho_{\mathrm{s}}+\rho_{s v}\right)}{\mathrm{d} t}-\frac{\mathrm{d}\left(\rho_{v}+\rho_{v \mathrm{~s}}\right)}{\mathrm{d} t}\right]_{t r},
$$

where $\dot{\rho}_{g, t r}$ denotes the change of $\rho_{g}$ because of the transformation of $\left(\rho_{s}+\rho_{s v}\right)$ and $\left(\rho_{v}+\rho_{v s}\right)$ to each other.

According to this model, $\rho_{g}$ is a function of $R V_{g},\left(\rho_{s}+\rho_{s v}\right)$ and $\left(\rho_{v}+\rho_{v s}\right)$, i.e. $\rho_{g}=\rho_{g}\left(R, V_{g},\left(\rho_{s}+\rho_{s v}\right),\left(\rho_{v}+\rho_{v s}\right)\right)$. Thus,

$$
\frac{\mathrm{d} \rho_{g}}{\mathrm{~d} t}=\frac{\partial \rho_{g}}{\partial R} \frac{\partial R}{\partial t}+\frac{\partial \rho_{g}}{\partial V_{g}} \frac{\partial V_{g}}{\partial t}+\left(\frac{\mathrm{d} \rho_{g}}{\mathrm{~d} t}\right)_{t r}
$$




$$
\dot{\rho}_{g, R V}=\dot{\rho}_{g}-\dot{\rho}_{g, t r}, \dot{\rho}_{g, R V} \equiv \frac{\partial \rho_{g}}{\partial R} \frac{\partial R}{\partial t}+\frac{\partial \rho_{g}}{\partial V_{g}} \frac{\partial V_{g}}{\partial t} .
$$

From (4.5)-(4.6) we have

$$
\dot{\rho}_{g, R V}=\dot{\rho}_{g}-\dot{\rho}_{g, t r}=-3\left(\rho_{g}+p_{g}\right) \dot{R} / R-\dot{V}_{g} .
$$

This is because $\dot{R}$ and $\dot{V}_{g}$ determine only $\dot{\rho}_{g, R V}$, but do not determine $\dot{\rho}_{g}$. It is obvious that (4.6) can be derived from (4.5) and (4.15). Considering the two equations (4.5)-(4.6), the equation determining $\dot{\rho}_{g, t r}$ (see section $6 B), p_{s}=p_{s}\left(\rho_{s}\right)$ and $p_{v}=p_{v}\left(\rho_{v}\right)$ or $p_{g}=p_{g}\left(\rho_{g}\right)$, we can determine the five variables $R$, $\left(\rho_{s}+\rho_{s v}\right),\left(\rho_{v}+\rho_{v s}\right), p_{s}$ and $p_{v}$, and further can determine $\rho_{s v}$ by (4.10).

When $T_{s}$ and $T_{v}$ are low or $T_{s}$ and $T_{v}$ are high enough so that $\rho_{s} \sim \rho_{v}$, the transformation of $\rho_{s}$ and $\rho_{v}$ may be neglected. Thus, $\dot{\rho}_{g, t r}=0$ and $\dot{\rho}_{g, R V} \simeq \dot{\rho}_{g}$, i.e.

$$
\dot{\rho}_{g}=-3\left(\rho_{g}+p_{g}\right) \dot{R} / R-\dot{V}_{g} .
$$

Pressure density is a function of masses of particles and temperature, i.e. $p=p(m, T)$. Let $p_{s}=l_{s} \rho_{s}$ and $p_{v}=l_{v} \rho_{v}$. In the S-breaking, from (4.16) we have

$$
\frac{\mathrm{d}\left(\rho_{s} R^{3\left(1+l_{s}\right)}\right)}{R^{3\left(1+l_{s}\right)} \mathrm{d} t}-\frac{\mathrm{d}\left(\rho_{v} R^{3\left(1+l_{v}\right)}\right)}{R^{3\left(1+l_{v}\right)} \mathrm{d} t}=-\dot{V}_{g} .
$$

It is obvious that when $\dot{V}_{g} \sim 0$, the solution of (4.14) is

$$
\rho_{s} R^{3\left(1+l_{s}\right)}=\rho_{s 0} R_{0}^{3\left(1+l_{s}\right)} \text {. and } \rho_{v} R^{3\left(1+l_{v}\right)}=\rho_{v 0} R_{0}^{3\left(1+l_{v}\right)} .
$$

In general, $l_{s} \neq l_{v}$.

In order to determine the pressure at a given temperature, we divide the particles into three sorts according to their masses. The first sort is composed of such particles whose masses $m_{M}^{\prime} s$ satisfy $m_{M}>m_{p}$, here $m_{p}$ is the mass of a proton. The second sort of particles is composed of such particles whose masses $m_{l}^{\prime} s$ satisfy $m_{p} \gtrsim m_{l} \geq m_{e}$, here $m_{e}$ is the mass of an electron. The third sort is composed of photon-like particles whose masses $m_{\gamma}^{\prime} s$ satisfy $m_{e}>m_{\gamma} \geq 0$ and $p_{\gamma} / \rho_{\gamma} \simeq 1 / 3$. When $T \gg m_{p}, p_{M} / \rho_{M} \sim p_{l} / \rho_{l} \simeq 1 / 3$; When $m_{p}>$ $T>m_{e}, \quad p_{M} / \rho_{M} \sim 0, \quad p_{l} / \rho_{l}=l$ and $1 / 3>l>0$. When $m_{e}>T, \quad p_{M} / \rho_{M} \sim p_{l} / \rho_{l} \sim 0$. Thus, we have

$$
\rho_{g}=\rho_{\mathrm{Mg}}+\rho_{\mathrm{lg}}+\rho_{\gamma g} \text {. }
$$

In the S-breaking, $\rho_{M g}=\rho_{s M}-\rho_{v M}, \quad \rho_{l g}=\rho_{s l}-\rho_{v l}$. Considering all v-particles must be in $v$-SU(5) color singlets whose masses are not zero so that $p_{v \gamma}=0$, we have $\rho_{\gamma g}=\rho_{s \gamma}$.

When $T_{s}$ and $T_{v}$ are so large that all masses may be neglected ( $T_{s}$ and $T_{v} \gg m_{p}$ ), from (4.16) we have

$$
p_{g}=\rho_{g} / 3, \frac{\mathrm{d}\left(\rho_{g} R^{4}\right)}{R^{4} \mathrm{~d} t}=-\dot{V}_{g} .
$$

When $m_{M}>T_{s} \gtrsim T_{v}>m_{e}, \quad p_{M} / \rho_{M} \sim 0$. Letting $p_{l g}=l \rho_{l g}$, we have

$$
\frac{\mathrm{d}\left(\rho_{M g} R^{3}\right)}{R^{3} \mathrm{~d} t}+\frac{\mathrm{d}\left(\rho_{l g} R^{3(1+l)}\right)}{R^{3(1+l)} \mathrm{d} t}+\frac{\mathrm{d}\left(\rho_{\gamma g} R^{4}\right)}{R^{4} \mathrm{~d} t}=-\dot{V}_{g} .
$$

When $m_{e}>T_{s} \gtrsim T_{v}, \quad p_{M} / \rho_{M} \sim 0$ and $p_{l} / \rho_{l} \sim 0$, we have

$$
\frac{\mathrm{d}\left(\rho_{m g} R^{3}\right)}{R^{3} \mathrm{~d} t}+\frac{\mathrm{d}\left(\rho_{\gamma g} R^{4}\right)}{R^{4} \mathrm{~d} t}=-\dot{V}_{g} .
$$

where $\rho_{m g}=\rho_{M g}+\rho_{l g}$.

$$
\frac{\mathrm{d}\left(\rho_{s} R^{3\left(1+l_{s}\right)}\right)}{R^{3\left(1+l_{s}\right)} \mathrm{d} t}-\frac{\mathrm{d}\left(\rho_{v} R^{3\left(1+l_{v}\right)}\right)}{R^{3\left(1+l_{v}\right)} \mathrm{d} t}=-\dot{V}_{g} .
$$

It is obvious that when $\dot{V}_{g} \simeq 0$, (4.21) has such solutions in the following form, 


$$
\begin{aligned}
& \rho_{s M} R^{3}=\rho_{s M 0} R_{0}^{3}, \rho_{v M} R^{3}=\rho_{v M 0} R_{0}^{3} ; \\
& \rho_{s l} R^{3\left(1+l_{s}\right)}=\rho_{s l 0} R_{0}^{3\left(1+l_{s}\right)}, \rho_{v l} R^{3\left(1+l_{v}\right)}=\rho_{v l 0} R_{0}^{3\left(1+l_{v}\right)}, \\
& \rho_{s \gamma} R^{4}=\rho_{s \gamma 0} R_{0}^{4} .
\end{aligned}
$$

In contrast with the conventional theory, $\rho_{g}>0, \rho_{g}=0$ and $\rho_{g}<0$, and $w_{g}>0, w_{g}=0$ and $w_{g}<0$ are all possible in the present model, here $w_{g} \equiv p_{g} / \rho_{g}$. For example, when temperature is so low that $\rho_{\gamma g} / \rho_{m g} \sim 0$ and $p_{s M} \simeq p_{s l} \simeq 0$, we have $p_{m g} \simeq-p_{v l}<0$ and $w_{g}<0$.

\section{Temperature Effect}

The thermal equilibrium between the v-particles and the s-particles can be realized by only (2.10). The Higgs bosons $\Omega_{s}$ and $\Omega_{v}$ are hardly produced because their masses are all very big in low temperatures. Consequently, the interaction between the $v$-particles and the s-particles may be ignored so that there is no thermal equilibrium between the v-particles and the s-particles. Thus, when temperature is low, we should use two sorts of temperature $T_{v}$ and $T_{s}$ to describe the thermal equilibrium of $v$-matter and the thermal equilibrium of s-matter, respectively. Generally speaking, $T_{v} \neq T_{s}$. When temperature is high enough, e.g. $T_{s} \sim T_{c r}$, the masses of the Higgs particles originating from (2.10) are small so that $\rho_{s}$ and $\rho_{v}$ can transform from one to another by (2.10). In the case, $T_{v}=T_{s}$ is possible.

\subsection{Effective Potentials}

Influence of finite temperature on the Higgs potential in the present model are consistent with the conventional theory. When the finite temperature effect is considered, the Higgs potential at zero-temperature becomes effective potential.

For short, we consider only $\Omega_{a}$ and $\varphi_{a}, a=s$ or $v$. When $\chi_{a}$ is considered as well, the following inferences are still qualitatively valid. From (2.8) we take

$$
V_{\Omega s}\left(\Omega_{s}\right)=-\frac{\mu^{2}}{2} \Omega_{s}^{2}+\frac{\lambda}{4} \Omega_{s}^{4},
$$

to ignore the terms proportional to $\lambda^{n}(n>1)$, to consider the temperature effect, the effective potential approximate to 1-loop in flat space is [25]-[27]

$$
V_{\Omega s, e f f}^{(1) T}\left(\bar{\Omega}_{s}, T_{s}\right)=-\frac{1}{2}\left(\mu^{2}-\frac{\lambda}{4} T_{s}^{2}\right) \bar{\Omega}_{s}^{2}+\frac{\lambda}{4} \bar{\Omega}_{s}^{4}-\frac{\pi^{2}}{90} T_{s}^{4}+\frac{\mu^{2}}{24} T_{s}^{2} .
$$

Considering the contributions of the expectation values $v_{\Omega v}\left(T_{s}, T_{v}\right), \quad v_{\varphi s}\left(T_{s}, T_{v}\right)$ and $v_{\varphi v}\left(T_{s}, T_{v}\right)$ to $V_{\Omega s, \text { eff }}^{(1) T}\left(\bar{\Omega}_{s}, T_{s}\right)$, and ignoring the terms irrelevant to $\Omega_{s}$, we have

$$
\begin{gathered}
V_{\Omega s, e f f}^{(1) T}\left(\bar{\Omega}_{s}, T_{s}, T_{v}\right)=-\frac{1}{2} \mu_{s}^{2}\left(T_{s}, T_{v}\right) \bar{\Omega}_{s}^{2}+\frac{\lambda}{4} \bar{\Omega}_{s}^{4}, \\
v_{\Omega s}^{2}\left(T_{s}, T_{v}\right)=\mu_{s}^{2}\left(T_{s}, T_{v}\right) / \lambda, \\
\mu_{s}^{2}\left(T_{s}, T_{v}\right) \equiv \mu^{2}-\frac{\lambda}{4} T_{s}^{2}-\Lambda v_{\Omega v}^{2}\left(T_{s}, T_{v}\right)-\frac{15}{2}\left[p v_{\varphi v}^{2}\left(T_{s}, T_{v}\right)-w v_{\varphi s}^{2}\left(T_{s}, T_{v}\right)\right] .
\end{gathered}
$$

Similarly (5.1)-(5.5), from (2.9) we have

$$
\begin{gathered}
V_{\Omega v, e f f}^{(1) T}\left(\bar{\Omega}_{v}, T_{s}, T_{v}\right)=-\frac{1}{2} \mu_{v}^{2}\left(T_{s}, T_{v}\right) \bar{\Omega}_{v}^{2}+\frac{\lambda}{4} \bar{\Omega}_{v}^{4}, \\
v_{\Omega v}^{2}\left(T_{s}, T_{v}\right)=\mu_{v}^{2}\left(T_{s}, T_{v}\right) / \lambda, \\
\mu_{v}^{2}\left(T_{s}, T_{v}\right) \equiv \mu^{2}-\frac{\lambda}{4} T_{v}^{2}-\Lambda v_{\Omega s}^{2}\left(T_{s}, T_{v}\right)-\frac{15}{2}\left[p v_{\varphi s}^{2}\left(T_{s}, T_{v}\right)-w v_{\varphi v}^{2}\left(T_{s}, T_{v}\right)\right] .
\end{gathered}
$$

From (2.8) we take 


$$
V_{\varphi s}\left(\Phi_{s}\right)=\frac{1}{2}\left(p \Omega_{v}^{2}-w \Omega_{s}^{2}\right) \operatorname{Tr} \Phi_{s}^{2}+\frac{1}{4} a\left(\operatorname{Tr} \Phi_{s}^{2}\right)^{2}+\frac{1}{2} b \operatorname{Tr} \Phi_{s}^{4},
$$

ignoring the contributions of the Higgs fields and the fermion fields to one loop correction, and only considering the contribution of the gauge fields, when $\bar{\varphi}_{s} \ll k T$, here $k$ is the Boltzmann constant (here $k=1$ ), we get the effective potential approximate to 1-loop in flat space at finite-temperature [25]-[27]

$$
V_{\varphi s, e f f}^{(1) T}\left(\bar{\varphi}_{s}, T_{s}\right)=V\left(\bar{\varphi}_{s}\right)+B \bar{\varphi}_{s}^{4}\left(\ln \frac{\bar{\varphi}_{s}^{2}}{\sigma^{2}}-\frac{25}{6}\right)+C T_{s}^{2} \bar{\varphi}_{s}^{2}-\frac{\pi^{2}}{15} T_{s}^{4},
$$

where $B=\left(5625 / 1024 \pi^{2}\right) g^{4}$, and $\Phi_{s}=\operatorname{Diagonal}(1,1,1,-3 / 2,-3 / 2) \bar{\varphi}_{s}$. In general, $w \sim p<g^{4}<C=(75 / 16)(\mathrm{kg})^{2}$. We take $w=p$ for simplicity. Here $\sigma$ is a parameter at which the renormalization coupling-constant is defined.

Only considering the contribution of the expectation values of $\Omega_{s}$ and $\Omega_{v}$ to $V_{\varphi s, \text { eff }}^{(1) T}\left(\bar{\varphi}_{s}, T_{s}\right)$, taking $(15 / 16)(15 a+7 b)=(11 / 3) B$ and ignoring the terms irrelevant with $\bar{\varphi}_{v}$, from (2.8) and (5.8)-(5.9), we have

$$
\begin{aligned}
& V_{\varphi s, \text { eff }}^{(1) T}\left(\bar{\varphi}_{s}, T_{s}, T_{v}\right)=A_{s}^{2}\left(T_{s}, T_{v}\right) \bar{\varphi}_{s}^{2}+B \bar{\varphi}_{s}^{4}\left(\ln \frac{\bar{\varphi}_{s}^{2}}{\sigma^{2}}-\frac{1}{2}\right), \\
& A_{s}^{2}\left(\bar{\varphi}_{s}, T_{s}, T_{v}\right) \equiv \frac{15}{4} w\left(v_{\Omega v}^{2}\left(T_{s}, T_{v}\right)-v_{\Omega s}^{2}\left(T_{s}, T_{v}\right)\right)+C T_{s}^{2} .
\end{aligned}
$$

It is easily seen from (5.10) that $v_{\varphi s}^{2}\left(T_{s}, T_{v}\right)=\sigma^{2}$ when $A_{s}^{2}\left(\sigma, T_{s}, T_{v}\right)=0$.

Similarly, from (2.9) we have

$$
\begin{aligned}
& V_{\varphi v, e f f}^{(1) T}\left(\bar{\varphi}_{v}, T_{s}, T_{v}\right)=A_{v}^{2}\left(T_{s}, T_{v}\right) \bar{\varphi}_{v}^{2}+B \bar{\varphi}_{v}^{4}\left(\ln \frac{\bar{\varphi}_{v}^{2}}{\sigma^{2}}-\frac{1}{2}\right), \\
& A_{v}^{2}\left(\bar{\varphi}_{v}, T_{s}, T_{v}\right) \equiv \frac{15}{4} w\left(v_{\Omega s}^{2}\left(T_{s}, T_{v}\right)-v_{\Omega v}^{2}\left(T_{s}, T_{v}\right)\right)+C T_{v}^{2}
\end{aligned}
$$

When the masses of all particles may be neglected, $p_{g}=\rho_{g} / 3$ and $\rho_{a}=\left(\pi^{2} / 30\right) g_{a}^{*} T_{a}^{4}, \quad g_{a}^{*}=g_{a B}+(7 / 8) g_{a F}$. $g_{a}^{*}$ is the total number of spin states, and $g_{a b}$ and $g_{a F}$ are the total number of spin states of $a$-bosons and the total number of spin states of a-femions, respectively. $g_{s}^{*}=g_{v}^{*} \equiv g^{*}$, because s-particles and $v$-particles are symmetric. Considering (4.5)-(4.7), (4.9), (5.2) and (5.9) in the S-breaking, we have

$$
\begin{aligned}
\dot{R}^{2} & =1+\eta\left[\rho_{g}+V_{g, \text { eff }}\right] R^{2}, \\
\ddot{R} & =-\frac{\eta}{2}\left[\rho_{g}+3 p_{g}-2 V_{g, \text { eff }}\right] R, \\
\dot{\rho}_{g}= & -3\left(\rho_{g}+3 p_{g}\right) \frac{\dot{R}}{R}-\dot{V}_{g, \text { eff }}=-4 \frac{\dot{R}}{R} \rho_{g}-\dot{V}_{g, \text { eff }} \text { when } p_{g}=\rho_{g} / 3 . \\
V_{g, \text { eff }} & =V_{s, \text { eff }}-V_{v, \text { eff }}, \\
V_{s, \text { eff }} & =V_{\varphi s, \text { eff }}+V_{\Omega s, e f f}+V_{0}-\left(\frac{\pi^{2}}{90}+\frac{\pi^{2}}{15}\right) T_{s}^{4}, \\
V_{v, \text { eff }} & =-\left(\frac{\pi^{2}}{90}+\frac{\pi^{2}}{15}\right) T_{v}^{4} .
\end{aligned}
$$

\subsection{The Critical Temperature $T_{\varphi c r}$ and Substable States in the $S$-Breaking}

For short, we take $V_{\text {eff }} \equiv V_{\text {eff }}^{(1) T}$ in the following. We consider such a space-contracting stage in which $\rho_{s}>\rho_{v}$ and $T_{s}>T_{v}$ in the S-breaking. We will see that there are the critical temperatures $T_{r r}, T_{\varphi c r 1}$ and $T_{\varphi c r}$ and $T_{c r}>T_{\varphi c r 1}>T_{\varphi c r}$. For the effective potential, there still is the S-breaking, i.e. $\left\langle\omega_{s}\left(T_{s}, T_{v}\right)\right\rangle \neq 0$ and $\left\langle\omega_{v}\left(T_{s}, T_{v}\right)\right\rangle=0$ when $T_{s} \leq T_{c r}$ and $\left\langle\omega_{s}\left(T_{s}, T_{v}\right)\right\rangle=\left\langle\omega_{v}\left(T_{s}, T_{v}\right)\right\rangle=0$ when $T_{s}>T_{c r}$ by suitably choosing the 
parameters in the Higgs potential.

As mentioned before, there is the S-breaking in low temperatures. Talking $p=w$ for short, we have

$$
\begin{aligned}
& \mu_{s}^{2}\left(T_{s}\right) \equiv \mu^{2}-\frac{\lambda}{4} T_{s}^{2}+\frac{15}{2} w v_{\varphi s}^{2}\left(T_{s}, T_{v}\right), \\
& \mu_{v}^{2}\left(T_{s}, T_{v}\right) \equiv \mu^{2}-\frac{\lambda}{4} T_{v}^{2}-\Lambda v_{\Omega s}^{2}\left(T_{s}\right)-\frac{15}{2} w v_{\varphi s}^{2}\left(T_{s}\right), \\
& A_{s}^{2}\left(T_{s}\right) \equiv-\frac{15}{4} w v_{\Omega s}^{2}\left(T_{s}, T_{v}\right)+C T_{s}^{2}, \\
& A_{v}^{2}\left(T_{s}, T_{v}\right) \equiv \frac{15}{4} w v_{\Omega s}^{2}\left(T_{s}, T_{v}\right)+C T_{v}^{2} .
\end{aligned}
$$

Both $T_{s}$ and $T_{v}$ will rise as space contracts. We will see that $T_{s}=T_{v}$ is possible when $T_{s}<T_{c r}$.

From (5.10) and (5.22) we can determine the minimum $V_{\varphi s, e f f, \min }$. Let $A_{s}^{2}\left(T_{s}, T_{v}\right) \equiv-2 B \sigma^{2} x e^{x}$. It is obviously that $x=x\left(T_{s}, T_{v}\right)$. It is seen from (5.10) and (5.22) that there are absolute minimums when $T_{s}$ is low, i.e.

$$
\begin{aligned}
& \frac{\mathrm{d} V_{\varphi s, e f f}}{\mathrm{~d} \bar{\varphi}_{s}}=0, \frac{\mathrm{d}^{2} V_{\varphi s, e f f}}{\mathrm{~d} \bar{\varphi}_{s}^{2}}>0 \text {, when } \bar{\varphi}_{s}=0 \\
& \text { and } \bar{\varphi}_{s}^{2}=v_{\varphi s}^{2}\left(T_{s}, T_{v}\right)=\sigma^{2} e^{x}, x \geq-1, V_{\varphi s, e f f}\left(v_{\varphi s}\right)<V_{\varphi s, e f f}(0) .
\end{aligned}
$$

$x$ will decrease monotonously as $T_{s}^{2}$ increases and its lower limit is -1 .

There is the critical temperature $T_{\varphi c r}$ at which the minimum is degenerate, i.e.

$$
\begin{gathered}
V_{\varphi s, e f f, \min }=V_{\varphi s, e f f}\left(v_{s \varphi c r}, T_{\varphi c r}, T_{v \varphi c r}\right)=V_{\varphi s, e f f}\left(0, T_{\varphi c r}, T_{v \varphi c r}\right)=0, \\
\frac{\partial V_{\varphi s, e f f}}{\partial \bar{\varphi}_{s}}=2 v_{s \varphi c r}\left(A_{s}^{2}\left(T_{\varphi c r}, T_{v \varphi c r}\right)+2 B v_{s \varphi c r}^{2} \ln \frac{v_{s \varphi c r}^{2}}{\sigma^{2}}\right)=0, \\
v_{s \varphi c r}^{2}=\sigma^{2} e^{-1 / 2}, T_{\varphi c r}^{2}=\frac{(15 w / 4 \lambda) \mu^{2}+\left(B+225 w^{2} / 8 \lambda\right) \sigma^{2} e^{-1 / 2}}{C+15 w / 16}, \\
A_{s}^{2}\left(T_{\varphi c r}, T_{v \varphi c r}\right)=-\frac{15}{4} w v_{\Omega s}^{2}\left(T_{\varphi c r}, T_{v \varphi c r}\right)+C T_{\varphi c r}^{2}=B \sigma^{2} e^{-1 / 2} \equiv A_{c r}^{2}, \\
A_{v}^{2}\left(T_{\varphi c r}, T_{v \varphi c r}\right)=\frac{15}{4} w v_{\Omega s}^{2}\left(T_{\varphi c r}, T_{v \varphi c r}\right)+C T_{v \varphi c r},
\end{gathered}
$$

where $T_{v}=T_{v \varphi c r}$ when $T_{s}=T_{s \varphi c r} \equiv T_{\varphi c r}$.

There is the critical temperature $T_{\varphi c r 1}$ at which

$$
\begin{gathered}
\frac{\partial V_{\varphi s, e f f}\left(v_{s \varphi c r 1}\right)}{\partial \bar{\varphi}_{s}}=\frac{\partial^{2} V_{\varphi s, e f f}\left(v_{s \varphi c r 1}\right)}{\partial \bar{\varphi}_{s}^{2}}=0, \\
V_{\varphi s, e f f}\left(v_{s \varphi c r 1}\right)>V_{\varphi s, e f f}(0), \bar{\varphi}_{s}^{2}=v_{s \varphi c r 1}^{2}\left(T_{\varphi c r 1}, T_{v \varphi c r 1}\right)=\sigma^{2} e^{-1}, \\
A_{s}^{2}\left(T_{\varphi c r 1}, T_{v \varphi c r 1}\right)=2 B \sigma^{2} e^{-1} \equiv A_{c r 1}^{2}, \\
T_{\varphi c r 1}^{2}=\left[\frac{15 w}{4 \lambda} \mu^{2}+\left(2 B+\frac{225}{8} \frac{w^{2}}{\lambda}\right) \sigma^{2} e^{-1}\right]\left(C+\frac{15}{16} w\right)^{-1},
\end{gathered}
$$

where $T_{v}=T_{v \varphi c r 1}$ when $T_{s}=T_{\varphi c r 1}$.

Sum up, when $T_{s}<T_{\varphi c r}, \quad A_{s}^{2}<A_{c r}^{2}, \quad x>-1 / 2$ and $V_{\varphi s, e f f}\left(v_{\varphi s}, T_{s}, T_{v}\right)$ is the absolute minimum, i.e. $V_{\varphi s, \text { eff } \text {, min }}<V_{\varphi s, e f f}(0)$. There is such a $T_{s}^{\prime}<T_{\varphi c r}$ that $x\left(T_{s}^{\prime}, T_{v}^{\prime}\right)=0, \quad A_{s}\left(T_{s}^{\prime}, T_{v}^{\prime}\right)=0, \quad v_{\varphi s}^{2}\left(T_{s}^{\prime}, T_{v}^{\prime}\right)=\sigma^{2}$ and 
$V_{\varphi s, \text { eff } \text {, min }}=-B \sigma^{4} / 2$. When $T_{\varphi c r 1}>T_{s}>T_{\varphi c r}, \quad A_{c r}^{2}<A_{s}^{2}<A_{c r 1}^{2},-1 / 2>x>-1$, and $V_{\varphi s, \text { eff }}\left(v_{\varphi s}, T_{s}, T_{v}\right)$ is a relative minimum and larger than $V_{\varphi s, \text { eff }}(0)$, i.e. there are substable states when $T_{\varphi c r 1}>T_{s}>T_{\varphi c r}$. When $A_{s}^{2} \geq A_{c r 1}^{2}$, i.e. $T_{s} \geq T_{\varphi c r 1}$,

$$
V_{\varphi s, \text { eff }, \text { min }}\left(\bar{\varphi}_{s}\right)=V_{\varphi s, \text { eff }}(0),\left\langle\varphi_{s}\right\rangle=0 \text {. }
$$

In the case, there is no relative minimum, as shown in Figure 1.

Analogously to that $\left\langle\varphi_{s}\right\rangle=0$ when $A_{s}^{2} \geq A_{c r 1}^{2}$, it is seen from (5.12) that $\left\langle\varphi_{v}\right\rangle=0$ when $A_{v}^{2} \geq A_{c r 1}^{2}$.

When $A_{s}^{2}<2 B \sigma^{2} e^{-1}$, i.e. $T_{s}<T_{\varphi c r 1}, v_{\varphi s}^{2}\left(T_{s}, T_{v}\right)>0$. We can get the masses of $\Omega_{s}, \Omega_{v}, \varphi_{s}$ and $\varphi_{v}$ from (5.3), (5.6), (5.10), (5.12) and (5.20)-(5.23)

$$
\begin{aligned}
& m_{\Omega s}^{2}=2 \lambda v_{\Omega s}^{2}=2\left(\mu^{2}-\frac{\lambda}{4} T_{s}^{2}+\frac{15}{2} w v_{\varphi s}^{2}\right), \text { when } T_{s}<T_{c r}, \\
& m_{\Omega v}^{2}=-\mu^{2}+\frac{\lambda}{4} T_{v}^{2}+\Lambda v_{\Omega s}^{2}+\frac{15}{2} w v_{\varphi s}^{2}, \text { when } T_{s}<T_{c r}, \\
& m_{\varphi s}^{2}=-4 A_{s}^{2}\left(1+x^{-1}\right)=\left(15 w v_{\Omega s}^{2}-4 C T_{s}^{2}\right)\left(1+x^{-1}\right), \text { when } A_{s}^{2}<A_{c r 1}^{2}, \\
& m_{\varphi s}^{2}=-\frac{15}{2} w v_{\Omega s}^{2}+2 C T_{s}^{2}=2 A_{s}^{2}, \text { when } A_{s}^{2} \geq A_{c r 1}^{2}, \\
& m_{\varphi v}^{2}=\frac{15}{2} w v_{\Omega s}^{2}+2 C T_{v}^{2}, \text { when } T_{s}<T_{c r} .
\end{aligned}
$$

\subsection{The Critical Temperature $T_{c r}$}

It is easily seen from (5.3)-(5.7) that $v_{\Omega s}=0$ when $\mu_{s}^{2}\left(T_{s}, T_{v}\right) \leq 0$, and $v_{\Omega v}=0$ when $\mu_{v}^{2}\left(T_{s}, T_{v}\right) \leq 0$. In the S-breaking, $v_{\varphi s}=0$ when $T_{s}>T_{\varphi c r 1}$ and $v_{\Omega v}=v_{\varphi v}=0$. Thus, from (5.3) we can determine the critical temperature $T_{c r}$ of $\Omega_{s}$.

$$
\begin{gathered}
\mu_{s}^{2}=\mu^{2}-\frac{\lambda}{4} T_{s}=0 \equiv \mu_{c r}^{2} \text { when } T_{s}=\frac{2 \mu}{\sqrt{\lambda}} \equiv T_{c r}>T_{\varphi c r 1}, \\
\mu_{s}^{2} \leq 0, v_{\varphi s}=0 \text { when } T_{s} \geq T_{c r} ; \mu_{s}^{2}>0, v_{\varphi s} \neq 0 \text { when } T_{s}<T_{c r} .
\end{gathered}
$$

Both $T_{s}$ and $T_{v}$ will rise as space contracts. Let $T_{v}=T_{v \Omega c r}$ when $T_{s}=T_{c r}$. We will see $T_{v \Omega c r}>T_{c r}$ in the following. It is easily seen from (5.5) and (5.41) when $T_{s}$ increases from $T_{s} \sim 0$ to $T_{s} \geq T_{c r}$ that $\mu_{s}^{2}\left(T_{s}, T_{v}\right)$ will decrease from $\mu^{2}+(15 / 2) w v_{\varphi s}^{2}$ to $\mu_{s}^{2}\left(T_{c r}\right) \leq 0$.

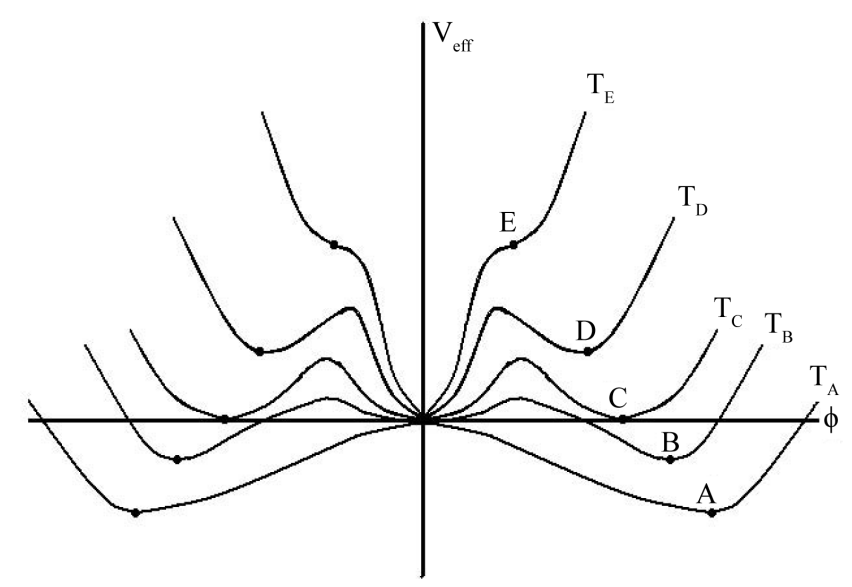

Figure 1. The states changes from $A$ to $E$ when $T_{S}$ rises from $T<$ $T_{\varphi c r}$ to $T_{\varphi c r 1}$. 


\section{Space Can Contract, But There Is No Singularity}

On the basis of the cosmological principle, if there is the space-time singularity, it may be a result of space contraction. Thus, we discuss the contracting process. From the contracting process we will see that there is no space-time singularity in present model.

\subsection{The Initial Condition and the Boundary Condition}

We consider the contracting process of the universe after expansion in the S-breaking. It is seen from (3.15) that in the case, $V_{g}=-V_{1}$. The initial condition is that at $t=t_{I}=0$,

$$
\begin{gathered}
\dot{R}^{2}\left(t_{I}\right)=1+\eta\left[\rho_{s}\left(t_{I}\right)-\rho_{v}\left(t_{I}\right)-V_{1}\right] R^{2}\left(t_{I}\right)=0, \rho_{s}\left(t_{I}\right)>\rho_{v}\left(t_{I}\right), \\
\ddot{R}\left(t_{I}\right)=-\frac{\eta}{2}\left[\rho_{s}\left(t_{I}\right)-\rho_{v}\left(t_{I}\right)+2 V_{1}\right] R\left(t_{I}\right)=\frac{1}{2}\left[\frac{1}{R\left(t_{I}\right)}-3 \eta V_{1} R\left(t_{I}\right)\right]<0, \\
p_{s}\left(t_{I}\right) \sim p_{v}\left(t_{I}\right) \sim 0, T_{s}\left(t_{I}\right) \sim T_{v}\left(t_{I}\right) \sim 0, R\left(t_{I}\right)=R_{\max } .
\end{gathered}
$$

It is obvious that $\rho_{s}\left(t_{I}\right)=\rho_{s \min }$ and $\rho_{v}\left(t_{I}\right)=\rho_{v \min }$. Space will contract when $t>t_{T}=0$, because $\dot{R}\left(t_{I}\right)=0$ and $\ddot{R}\left(t_{I}\right)<0$. We consider that the physical boundary condition of the Equations (5.14)-(5.15) should be

$$
\dot{R}=0 \text { when } R=R_{\min }>0 \text {. }
$$

In contrast with the conventional theory, there are such solutions which satisfy the boundary condition. This implies that there is no singularity in the model.

There is no singularity in the model [19] [20] as well. This is because $k$ is changeable in the model [19] [20]. It is possible that the model [19] [20] is better.

\subsection{Transformation of $\rho_{s}$ and $\rho_{v}$ from One to Another}

When both $T_{s}$ and $T_{v}$ are low, the transformation of $\rho_{s}$ and $\rho_{v}$ may be neglected because the masses of the Higgs particles are all very large. Consequently, $\rho_{s}$ and $\rho_{v}$ are independent of each other. When both $T_{s}$ and $T_{v}$ rise because space contracts, as mentioned before, the masses of the Higgs particles originating from the couplings (2.8)-(2.10) will reduce. Thus, the transformation of the $s$-Higgs particles and the $v$-Higgs particles by (2.10) is striking.

We discuss the transformation of $\rho_{s}$ and $\rho_{v}$ follows.

Let $\dot{\rho}_{g, t r 1}$ originate from decay the Higgs particles as $\Omega_{s} \rightarrow \Omega_{v}+\Omega_{v}$ and $\Omega_{s} \rightarrow \varphi_{v}+\varphi_{v}, \quad \dot{\rho}_{g \text {,tr } 2}$ originate

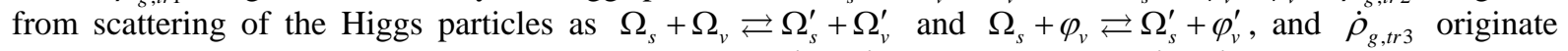
from scattering of the Higgs particles as $\Omega_{s}+\Omega_{s} \rightleftarrows \Omega_{v}^{\prime}+\Omega_{v}^{\prime}$ and $\Omega_{s}+\Omega_{s} \rightleftarrows \varphi_{v}^{\prime}+\varphi_{v}^{\prime}, \quad \dot{\rho}_{g, t r}$ may be written as

$$
\dot{\rho}_{g, t r}=2\left(\dot{\rho}_{s, t r}-\dot{\rho}_{v, t r}\right)=\dot{\rho}_{g, t r 1}+\dot{\rho}_{g, t r 2}+\dot{\rho}_{g, t r 3} \text {. }
$$

\subsection{1. $\rho_{\mathrm{g}}\left(t_{\varphi c r}\right)<0$ Is Possible When $T_{v} \leq T_{s}<T_{\varphi c r}$}

In the initial stage, temperature is low, i.e. $0 \leq T_{v} \lesssim T_{s}<T_{\varphi c r}, \quad\left\langle\omega_{s}\left(T_{s}\right)\right\rangle \sim\left\langle\omega_{s}(0)\right\rangle \neq 0$ and $\left\langle\omega_{v}\right\rangle=0$. Thus, $S U_{S}(5)$ breaks to $S U_{S}(3) \times U_{S}(1)$ when $T_{s} \lesssim T_{\chi c r}$, then to $S U_{S}(3) \times S U_{S}(2) \times U_{S}(1) \quad\left(v_{\Omega s} \neq 0, v_{\varphi s} \neq 0\right.$ and $\left.v_{\chi s}=0\right)$ when $T_{\chi c r}<T_{s}<T_{\varphi c r}$. When $T_{s} \geq T_{\varphi c r 1}$, $S U_{S}(5)$ symmetry holds $\left(\left|v_{\Omega s}\right| \geq 0, v_{\varphi s}=v_{\chi s}=0\right)$. $\left\langle\omega_{v}\right\rangle=0$ and $S U_{V}(5)$ hold all the time. Here $T_{\chi c r}$ is such a temperature that $\left\langle\chi_{s}\right\rangle=0$ when $T_{s} \geq T_{\chi c r}$ and $\left\langle\chi_{s}\right\rangle \neq 0$ when $T_{s}<T_{\chi c r}$.

When $T_{s}<T_{\varphi c r}$, the masses of the Higgs particles are large and the number of the Higgs particles is little. In the case, $V_{g} \sim 0$. Thus, (4.18) holds so that the transformation of $\rho_{s}$ and $\rho_{v}$ may be neglected. When $T_{s}<T_{\varphi c r}$, the $s$-particles must form celestial bodies with their large masses so that $p_{s}\left(T_{s}\right) / \rho_{s}\left(T_{s}\right) \sim 0$ and $\rho_{s}(t)=\rho_{s 0} R_{0}^{3} / R^{3}(t)$. However, the $v$-particles must be in $S U_{V}(5)$ color singlets so that

$0<p_{v}\left(T_{v}\right) / \rho_{v}\left(T_{v}\right)=l_{v} \lesssim 1 / 3$ and $\rho_{v}(t)=\rho_{v 0} R_{0}^{3\left(1+l_{v}\right)} / R^{3\left(1+l_{v}\right)}(t)$. It is obvious that the less the masses of the color singlets are, the larger $l_{v}$ is. Consequently,

$$
\rho_{v}(t) / \rho_{s}(t)=\left(\rho_{v 0} / \rho_{s 0}\right)\left(R_{0} / R(t)\right)^{3 l_{v}}>\left(\rho_{v 0} / \rho_{s 0}\right)
$$


and it is possible that $\rho_{v}\left(t_{1}\right) / \rho_{s}\left(t_{1}\right)=1$ when $t_{\varphi c r}>t_{1}>t_{I}$, here $t=t_{\varphi c r}$ when $T_{s}=T_{\varphi c r}$. In the case, there are such solutions satisfying (6.4) (see the discussion below, $t_{2} \rightarrow t_{1}$ in (6.13)-(6.15).

\subsubsection{A Super-Heating Process}

As mentioned in the preceding section, there are the substable states. Consequently, the universe will be in the substable states when $T_{s}$ rises from $T_{\varphi c r}$ to $T_{\varphi c r 1}$. The states changes from $A$ to $E$ when $T_{s}$ rises from $T<T_{\varphi c r}$ to $T_{\varphi c r 1}$, as shown by Figure 1 . Thus, there still are $\left\langle\omega_{s}\right\rangle \neq 0$ and $\dot{V}_{g, \text { eff }}>0$ although

$T_{\varphi c r} \leq T_{s}<T_{\varphi c r 1}$. It is seen that the contracting process is a super-heating process when $T_{\varphi c r} \leq T_{s}<T_{\varphi c r 1}$. The substable states are not stable. A substable state can transit to the stable state with $V_{\text {seff }}\left(\bar{\varphi}_{s}=0\right)=0$.

$$
\rho_{g}\left(T_{c r}\right)<0 \text { when } T_{\varphi c r 1}<T_{s}<T_{c r} \text {, even if } \rho_{g}\left(t_{\varphi c r 1}\right) \geq 0 \text {. }
$$

(1) The effective masses of the Higgs particles and the transformation of $\rho_{s}$ and $\rho_{v}$.

If $T_{c r}>T_{v} \geq T_{s}>T_{\varphi c r 1}$, there must be $\rho_{v} \geq \rho_{s}, V_{0}>V_{g, \text { eff }}\left(T_{s}\right)>0$ and $\dot{V}_{g, \text { eff }}\left(T_{s}\right)>0$. Thus, it is easily proved that there must be such a solution satisfying (6.4) in this case. Hence we consider such a contracting process when $T_{c r}>T_{s}>T_{v}, T_{s}>T_{\varphi c r 1}$ and $\rho_{g}\left(t_{\varphi c r 1}\right)>0$.

The masses containing the temperature effect are called effective masses.

The effective masses of $\Omega_{s}$ and $\Omega_{v}$ are important for $\dot{\rho}_{v, t e}$ and $\dot{\rho}_{s, t e}$. As mentioned before, $v_{\varphi s}=v_{\chi s}=0, \quad v_{\Omega s} \neq 0$ and $\left\langle\omega_{v}\right\rangle=0$, and both $S U_{S}(5)$ and $S U_{V}(5)$ hold when $T_{c r}>T_{s}>T_{v}$ and $T_{s}>T_{\varphi c r 1}$. From (5.35)-(5.36) we have

$$
\begin{aligned}
m_{\Omega v}^{2} & =-\mu_{v}^{2}=\frac{1}{2}\left(\frac{\Lambda}{\lambda}-1\right) m_{\Omega s}^{2}-\frac{\lambda}{4}\left(T_{s}^{2}-T_{v}^{2}\right) \\
& =\left(\frac{\Lambda}{\lambda}-1\right) \mu^{2}-\frac{\Lambda}{4} T_{s}^{2}+\frac{\lambda}{4} T_{v}^{2} \equiv\left(N m_{\Omega s}\right)^{2}, T_{s}>T_{v},
\end{aligned}
$$

It is seen from (6.7) that there must be such a $T_{s 1}$ that $m_{\Omega s}\left(T_{s 1}, T_{v 1}\right)>0, \quad m_{\Omega v}\left(T_{s 1}, T_{v 1}\right)=0$ because $T_{v}<T_{s}$ in this case. Here $T_{v}=T_{v 1}$ when $T_{s}=T_{s 1}$ and $T_{v 1}<T_{s 1} \cdot m_{\varphi s}\left(T_{s 1}, T_{v 1}\right)>0$ and $m_{\varphi v}\left(T_{s 1}, T_{v 1}\right)>0$ because $v_{\Omega s} \neq 0$. Thus, there must be such a $T_{s 2}$ that $m_{\varphi s}\left(T_{s 1}, T_{v 1}\right)$ and $m_{\Omega s}\left(T_{s 2}, T_{v 2}\right) \geq 2 m_{\Omega v}\left(T_{s 2}, T_{v 2}\right) \gtrsim 0$, here $T_{v 2}<T_{s 2}<T_{s 1}$ and $T_{v}=T_{v 2}$ when $T_{s}=T_{s 2}$. Consequently, there are such decays $\Omega_{s} \rightarrow \Omega_{v}+\Omega_{v}$ and $\Omega_{s} \rightarrow \varphi_{v}+\varphi_{v}$ so that $\rho_{s}$ can transform to $\rho_{v}$ and $T_{s}-T_{v}$ decreases, even if $m_{\varphi s} \lesssim m_{\Omega v}$. Consequently, there must is

$$
\dot{\rho}_{g, t e 1} \propto-2_{i}\left(n_{i s} \Gamma_{s i}\left(T_{s}\right) T_{s}-n_{i v} \Gamma_{v i}\left(T_{v}\right) T_{v}\right)<0, i=\Omega, \varphi, \chi,
$$

where $n_{i a}$ is the number density of the $i$-th sort of a-Higgs particles, $a=s, v$, and $\Gamma_{a i}\left(T_{a}\right)$ is the decay rate of the $i$-th sort of $a$-Higgs particles to the $b$-Higgs particles at the temperature $T_{a}, b=s, v$ and $b \neq a$. Here (6.5) is considered so that the factor $(-2)$ emerges in (6.8).

(2) $\dot{\rho}_{g, t r 2}<0$ and $\dot{\rho}_{g, t r 3}<0$ when $T_{s}>T_{v}$.

When both $m_{\Omega s}$ and $m_{\Omega v}$ are small (i.e. when $T_{c r}>T_{s}>T_{v}$ and $T_{s}>T_{\varphi c r 1}$ ), it is striking that such reactions as $\Omega_{s}+\Omega_{v} \rightleftarrows \Omega_{s}^{\prime}+\Omega_{v}^{\prime}, \quad \Omega_{s}+\varphi_{v} \rightleftarrows \Omega_{s}^{\prime}+\varphi_{v}^{\prime}, \quad \Omega_{s}+\Omega_{s} \rightleftarrows \Omega_{v}+\Omega_{v}$, and $\varphi_{s}+\varphi_{s} \rightleftarrows \Omega_{v}+\Omega_{v}$ etc. due to (2.10). Considering $T_{s}>T_{v}$, we have

$$
\begin{aligned}
& \dot{\rho}_{g, t r 2} \propto-2 \sum_{j}\left[n_{\Omega s} n_{j v} v_{\Omega s, j v} \sigma_{\Omega s, j v}\left(T_{s}, T_{v}\right)-n_{\Omega v} n_{j s} v_{\Omega v, j s} \sigma_{\Omega v, j s}\left(T_{s}, T_{v}\right)\right]\left(T_{s}-T_{v}\right)<0, \\
& \dot{\rho}_{g, t r 3} \propto-2 \sum_{i, j}\left[n_{i s}^{2} v_{i s} \sigma_{i s}\left(T_{s}\right) T_{s}-n_{i v}^{2} v_{i v} \sigma_{i v}\left(T_{v}\right) T_{v}\right]<0, i, j=\Omega, \varphi, \chi,
\end{aligned}
$$

where $\sigma_{\Omega s, j v}\left(\sigma_{\Omega v, j s}\right)$ is a scattering cross section of a $\Omega_{s}\left(\Omega_{v}\right)$ particle and a $\omega_{j v}\left(\omega_{j s}\right)$ particle, $v_{\Omega s, j v}$ $\left(v_{\Omega v, j s}\right)$ is a relative velocities of a $\Omega_{s}\left(\Omega_{v}\right)$ particle to a $\omega_{j v}\left(\omega_{j s}\right)$ particle, and $v_{i s}\left(v_{i v}\right)$ is a relative velocities of two $\omega_{i s}\left(\omega_{j v}\right)$ particles. (6.5) is considered so that the factor $(-2)$ emerges in (6.9)-(6.10). $\omega_{j v}$ $\left(\omega_{j s}\right)$ is the $j$-th sort of $v$-Higgs (s-Higgs) particles.

(3) $\dot{\rho}_{g, t r}<0$ when $T_{c r}>T_{s}>T_{v}$ and $T_{s}>T_{\varphi c r 1}$

It is obvious that the larger $T_{s}$ is, the less $\left|v_{\Omega s}\right|$ is and $v_{\Omega s}=0$ when $T_{s}=T_{c r}$. The larger $T_{s}$ is, the less the masses of all Higgs particles originating from the couplings (2.8)-(2.10) are. Thus, the masses of all Higgs particles originating from the couplings (2.8)-(2.10) are very small when $T_{s}>T_{\varphi c r 1}$. 
The masses of all gauge bosons and fermions are zero when $v_{\varphi s}=v_{\chi s}=0, v_{\Omega s} \neq 0$ and $\left\langle\omega_{\nu}\right\rangle=0$. Thus, the a-Higgs particles and the a-gauge bosons or the a-ferminos can transform from one to another by the $S U_{A}(5)$ couplings, here $a=s, v$ and $A=S, V$. Thus, the number density of the a-Higgs particles is large. The transformation of s-Higgs particles and the v-Higgs particles from one to another is striking when $T_{c r}>T_{s}>T_{v}$ and $T_{s}>T_{\varphi c r 1}$.

It is seen from the above mentioned and (6.8)-(6.10) that there must be

$$
\dot{\rho}_{g, t r}<0
$$

when $T_{c r}>T_{s}>T_{v}$ and $T_{s}>T_{\varphi c r 1}$. In the case, $\rho_{g}$ and $\left(T_{s}-T_{v}\right)$ must decrease so that $\dot{R} / R$ decreases.

(4) $\rho_{g}\left(t_{c r}\right)<0$.

Because of the symmetry of the s-particles and the v-particles, $g_{s}^{*}=g_{v}^{*} \equiv g^{*}$, here $g_{s}^{*}\left(g_{v}^{*}\right)$ is the spinfreedom of the s-particles (the $v$-particles). When $T_{c r}>T_{s} \geq T_{v}>T_{\varphi c r 1}, \quad \rho_{s}=\left(\pi^{2} / 30\right) g^{*} T_{s}^{4}$ and

$\rho_{v}=\left(\pi^{2} / 30\right) g^{*} T_{v}^{4}$. In contrast with the contracting process $(\dot{R}<0), \quad \dot{\rho}_{g, t r}$ causes $\rho_{g}$ and $T_{s}-T_{v}$ to decrease and the thermal equilibrium of s-matter and $v$-matter.

When $T_{c r}>T_{s}>T_{v}$ and $T_{s}>T_{\varphi c r 1}, \quad \rho_{s}$ is very large and the masses of all particles are so small that they may be neglected. Consequently, $\left|\dot{\rho}_{g, t r}\right| \gg\left|\left(\rho_{g}+p_{g}\right)(\dot{R} / R)\right|$ so that

$$
\dot{\rho}_{g}(t)=\dot{\rho}_{g, t r}-3\left(\rho_{g}+p_{g}\right)-\dot{V}_{g, e f f}<0,
$$

and there is such a moment $t_{2}$ at which

$$
\rho_{g}\left(t_{2}\right)=\rho_{g}\left(t_{\varphi c r 1}\right)+\int_{t_{\varphi c r 1}}^{t_{2}} \mathrm{~d} t \dot{\rho}_{g}(t)=0, T_{s}\left(t_{2}\right)=T_{v}\left(t_{2}\right)<T_{c r} .
$$

When $T_{s}=T_{v}, \quad \dot{\rho}_{g, t r} \sim 0$ and $m\left(\omega_{s}\right) \sim m\left(\omega_{v}\right) \neq 0$, but $m\left(\omega_{s}\right)$ and $m\left(\omega_{v}\right)$ are very small so that they may be neglected. In this case, (4.5)-(4.6) and (4.12) reduce to

$$
\begin{aligned}
& \dot{R}^{2}\left(t_{2}\right)=1+\eta V_{g, \text { eff }}\left(t_{2}\right) R^{2}\left(t_{2}\right), \\
& \ddot{R}\left(t_{2}\right)=\eta V_{g, \text { eff }}\left(t_{2}\right) R\left(t_{2}\right), \dot{\rho}_{g}\left(t_{2}\right)=-\dot{V}_{g, \text { eff }}\left(t_{2}\right)<0 .
\end{aligned}
$$

Thus, space will contract with a deceleration. Let $t=t_{c r}$ when $T_{s}=T_{c r}$, considering $\dot{V}_{g}>0$ because $\left|\left\langle\Omega_{s}\right\rangle\right|$ decreases due to space contraction, we have

$$
\rho_{g}\left(t_{c r}\right)=\int_{t_{2}}^{t_{c r}} \mathrm{~d} t\left[-3\left(\rho_{g}+p_{g}\right)-\dot{V}_{g, e f f}\right]<0, T_{v}>T_{s}=T_{c r} .
$$

\subsection{3. $\left\langle\omega_{v}\right\rangle=0$ and $m_{\Omega v} \geq 0$ in the S-Breaking for All Time}

We see from the discussion above that $m_{\Omega v} \geq 0$ can hold when $T_{c r} \geq T_{s} \geq T_{v}$. This is because

$m_{\Omega v}=m_{\Omega v}\left(T_{s}, T_{v}\right)$ is a continuous function of $T_{s}$ and $T_{v}$. If $m_{\Omega v}<0$ was arrived at some a time $t^{\prime}$, there must be such a time $t^{\prime \prime}<t^{\prime}$ so that $m_{\Omega v}\left(t^{\prime \prime}\right)=0$. When $m_{\Omega v}\left(t^{\prime \prime}\right) \sim 0$, the transformation of $\rho_{s}$ to $\rho_{v}$ must be striking so that $\left(T_{s}-T_{v}\right)$ decreases to 0 and $m_{\Omega v}$ will increase from $m_{\Omega v}=0$ to $m_{\Omega v}(t)>0$ when $t>t^{\prime}$. Hence $m_{\Omega v}<0$ cannot occur so that the S-breaking can hold all the time for the effective potential $V_{g, e f f}$.

\subsection{There Is No Singularity of Space-Time in the Present Model}

From (6.15) we see when $t \geq t_{c r}$,

$$
\begin{aligned}
& \left\langle\omega_{s}\right\rangle=\left\langle\omega_{v}\right\rangle=0, V_{g, \text { eff }}=V_{0}, \dot{V}_{g, \text { eff }}=0, \\
& \dot{R}^{2}=1+\eta\left(\rho_{g}+V_{0}\right) R^{2}, \quad \rho_{g}<0, \\
& \ddot{R}=-\eta\left(\rho_{g}-V_{0}\right) R, \quad \dot{\rho}_{g}=-4 \rho_{g} \frac{\dot{R}}{R} .
\end{aligned}
$$

Here $p_{g}=\rho_{g} / 3$ is considered. In the case, space can continue to contract, but there must be such a moment 
$t_{F}$ at which

$$
\begin{gathered}
\dot{R}\left(t_{F}\right)=\dot{\rho}_{g}\left(t_{F}\right)=0, \ddot{R}\left(t_{F}\right)=-\eta\left(\rho_{g}\left(t_{F}\right)-V_{0}\right) R\left(t_{F}\right)>0, \\
R\left(t_{F}\right)=R_{\min }=-\frac{1}{\eta\left(\rho_{g}\left(t_{F}\right)+V_{0}\right)}>0 .
\end{gathered}
$$

This is because $\rho_{g}<0$ and $\rho_{g} \propto R^{-4}$ when $t \geq t_{c r}$. It is easily seen that

$$
\begin{aligned}
& T_{s}\left(t_{F}\right)=T_{s \max }, T_{v}\left(t_{F}\right)=T_{v \max }=T_{\max }>T_{s \max }, \\
& \rho\left(t_{F}\right)=\rho_{\max }=\left(\pi^{2} / 30\right) g^{*}\left(T_{v \max }^{4}+T_{s \max }^{4}\right) .
\end{aligned}
$$

$T_{\max }$ and $\rho_{\max }$ are the highest temperature and the largest energy density in the universe, respectively. According to the present model, $T_{\max }$ and $\rho_{\max }$ must exist. We will see that $t_{F}$ is just the final moment $t_{S F}$ of the $S$-world and the initial moment $t_{V I}$ of the $V$-world as well, i.e. $t_{F}=t_{S F}=t_{V I}$.

In summary, there are $R_{\min }, T_{\max }$ and $\rho_{\max }$ which are finite for the contracting process. Because of the cosmological principle, all $\rho_{s}, \rho_{v}, \rho_{g}, V_{s}, V_{v}, V_{g}$ and $p_{g} \leq \rho_{g} / 3$ are finite. Consequently $T_{s \mu \nu}$, $T_{v \mu v}, \quad T_{S g \mu v}$ and $T_{V g \mu v}$ must be finite. On the other hand, because of the cosmological principle, it is obvious that if there is no space contraction, the physical quantities must be finite as well. Substituting the finite $T_{S g \mu \nu}$ or $T_{V g \mu v}$ into the Einstein field equation, we see that $R_{\mu v}$ and $g_{\mu v}$ must be finite. Consequently, there is no singularity of space-time in the present model.

\subsection{The Result above Is Not Contradictory to the Singularity Theorems}

We first intuitively explain the reasons that there is no space-time singularity. It has been proved that there is space-time singularity under certain conditions [1]. These conditions fall into three categories. First, there is the requirement that gravity shall be attractive. Secondly, there is the requirement that there is enough matter present in some region to prevent anything escaping from that region. The third requirement is that there should be no causality violations.

Hawking considers it is a reasonable the first condition that $\rho_{g}=\rho>0$ and $p_{g} \geq 0$ [1]. But this conjecture is not valid in the present model, because all $\rho_{g}=\rho_{s}-\rho_{v}>0,=0$ or $<0$ are possible. $T_{\mu v}$ in general relativity is equivalent with $T_{g \mu v}$ in the present model. In contrast with $T_{\mu v}, T_{g \mu v}$ is not the energymomintum tensor so that it does not satisfy the energy condition due to the conjecture 1 . On the other hand, $E_{s}$ and $E_{v}$ can transform from one to other, especially when temperature $T \sim T_{c r}$. Hence the premise of the singularity theorems does not hold so that the the singularity theorems are invalid in the present model.

As mentioned above, there must be $\rho_{g}<0$ when $T \geq T_{c r}$. It is seen that $\rho_{g}$ does not only stop increasing, but also decreases from $\rho_{g}(t)>0 \quad\left(t<t_{2}\right)$ to $\rho_{g}\left(t_{2}\right)=0$ and finally to $\rho_{g}(t)<0$ when $t>t_{2}$. Hence the second condition of the singularity theorem is violated.

The key of non-singularity is conjecture 1, i.e. $\rho_{s g}=-\rho_{v g}$ when $\rho_{s}=\rho_{v}$ and $\rho_{s}$ and $\rho_{v}$ can transform from one to another.

We explain the reasons that there is no space-time singularity from the Hawking theorem as follows. S.W. Hawking has proven the following theorem [1].

The following three conditions cannot all hold:

(a) every inextendible non-spacelike geodesic contains a pair of conjugate point;

(b) the chronology condition holds on $\mu$;

(c) there is an achronal set $\mathfrak{T}$ such that $E^{+}(\mathfrak{T})$ or $E^{-}(\mathfrak{T})$ is compact.

The alternative version of the theorem can obtained by the following two propositions.

Proposition 1 [1]:

If $R_{a b} V^{a} V^{b} \geq 0$ and if at some point $p=\gamma\left(s_{1}\right)$ the tidal force $R_{a b c d} V^{c} V^{d}$ is non-zero, there will be values $s_{0}$ and $s_{2}$ such that $q=\gamma\left(s_{0}\right)$ and $r=\gamma\left(s_{2}\right)$ will be conjugate along $\gamma(s)$, providing that $\gamma(s)$ can be extended to these values.

Proposition 2 [1]:

If $R_{a b} V^{a} V^{b} \geq 0$ everywhere and if at $p=\gamma\left(v_{1}\right), \quad K^{a} K^{b} K_{[a} R_{b] c d[e} K_{f]}$ is non-zero, there will be $v_{0}$ and $v_{2}$ such that $q=\gamma\left(v_{0}\right)$ and $r=\gamma\left(v_{2}\right)$ will be conjugate along $\gamma(v)$ provided that $\gamma(v)$ can be extended 
to these values.

An alternative version of the above theorem is as following.

Space-time $(\mu, g)$ is not timelike and null geodesically complete if:

(1) $R^{a b} K_{a} K_{b} \geq 0$ for every non-spacelike vector $\boldsymbol{K}$.

(2) The generic condition is satisfied, i.e. every non-spacelike geodesic contains a point at which $K_{[a} R_{b] c d[e} K_{f]} K^{c} K^{d} \neq 0$, where $K$ is the tangent vector to the geodesic.

(3) The chronology condition holds on $\mu$ (i.e. there are no closed timelike curves).

(4) There exists at least one of the following:

(A) a compact achronal set without edge,

(B) a closed trapped surface,

(C) a point $p$ such that on every past (or every future) null geodesic from $p$ the divergence $\hat{\vartheta}$ of the null geodesics from $p$ becomes negative (i.e. the null geodesics from $p$ are focussed by the matter or curvature and start to reconverge).

In fact, $R_{a b}$ is determined by the gravitational energy-momentum tensor $T_{g a b}$. According to the conventional theory, $T_{g a b}=T_{a b}$ so that the above theorem holds.

In contrast with the conventional theory, according to conjecture 1 ,

$$
S_{g \mu v} \equiv T_{g \mu v}-\frac{1}{2} g_{\mu v} T_{g}=\left(T_{v \mu v}-T_{s \mu v}\right)-\frac{1}{2} g_{\mu v}\left(T_{v}-T_{s}\right),
$$

$S_{g 00}>0,=0$ and $<0$ are all possible. Thus, although the strong energy condition still holds, i.e.

$$
\left[\left(T_{s}^{a b}+T_{v}^{a b}\right)-\frac{1}{2} g^{a b}\left(T_{s}+T_{v}\right)\right] K_{a} K_{b} \geq 0,
$$

the conditions of propositions 1 and 2 and condition (1) no longer hold, because the gravitational mass density $\rho_{g}$ determines $R_{\mu v}$ and $\rho_{g}=\rho_{v}-\rho_{s} \neq \rho_{v}+\rho_{s}=\rho$. Hence (a) and (c) do not hold, but (b) still holds, and $\mu$ is timelike and null geodesically complete.

\section{Space Inflation}

\subsection{Space Inflation}

When $T_{s} \geq T_{c r}, \quad\left\langle\omega_{s}\right\rangle=\left\langle\omega_{v}\right\rangle=0$ and $\dot{V}_{g, \text { eff }}=0$. We call such a state in which $\left\langle\omega_{s}\right\rangle=\left\langle\omega_{v}\right\rangle=0$ the most symmetric state. In this state the $S U_{S}(5) \times S U_{V}(5)$ symmetry holds strictly and the s-particles and the $v$-particles are symmetric.

When $R=R_{\min }, \quad \dot{R}=0$ and $\ddot{R}>0$. Hence space will expand when $t>t_{F}$.

Consider the initial stage of expansion in which $\rho_{\min }<\rho_{g}<0, T_{s}<T_{v}$ and $V_{g, \text { eff }}=V_{0} . \dot{R}(t) \gtrsim 0$ is small due to $\dot{R}\left(t_{F}\right)=0$. On the other hand, $\dot{\rho}_{g, t r 1}=0$ because $\left\langle\omega_{s}\right\rangle=\left\langle\omega_{v}\right\rangle=0$ and the s-particles and the $v$-particles are symmetric. $\dot{\rho}_{g, t r 2}>0$ and $\dot{\rho}_{g, t r 3}>0$ because (6.5)-(6.6), $\rho_{s}<\rho_{v}$ and $T_{s}<T_{v}$. Hence $\dot{\rho}_{g, t r}>0$. In the case, (4.5)-(4.6) and (4.15) become

$$
\begin{aligned}
& \dot{R}^{2}(t)=1+\eta\left(\rho_{g}+V_{0}\right) R^{2}(t)>0, \\
& \ddot{R}(t)=\eta\left(-\rho_{g}+V_{0}\right) R(t)>0, \\
& \dot{\rho}_{g}=\dot{\rho}_{g, t r}-4 \rho_{g}(\dot{R} / R)>0
\end{aligned}
$$

because $p_{g}=\rho_{g} / 3$ and $\rho_{\min }<\rho_{g}<0$. It is seen from (7.1)-(7.3) that there is such a time $t_{A}$ at which $\rho_{g}=0$. Furthermore, $\dot{\rho}_{g, t r}=0$ because $\rho_{s}=\rho_{v}$ and $T_{s}=T_{v}$. Thus, when $t \gtrsim t t_{A}$, (7.1)-(7.3) reduce to

$$
\dot{R}^{2}(t)=1+\eta V_{0} R^{2}(t), \ddot{R}(t)=\eta V_{0} R(t)>0, \dot{\rho}_{g}(t)=0 .
$$

Consequently, space inflation must occur

$$
R(t)=\sqrt{\frac{1}{\eta V_{0}}} \sinh H\left(t-t_{A}+\tau\right), H \equiv \sqrt{\eta V_{0}},
$$




$$
\begin{aligned}
& \sim \frac{1}{2} \exp H\left(t-t_{A}+\tau\right) \text {, when } H\left(t-t_{A}+\tau\right) \gg 1, \\
& R\left(t_{A}\right)=\sqrt{\frac{1}{\eta V_{0}}} \sinh H \tau .
\end{aligned}
$$

\subsection{The Process of Space Inflation}

Supposing $\lambda \sim g^{4}$ and $g^{2} \sim 4 \pi / 45$ for $S U(5)$, and considering $m\left(\Omega_{s}\right)=\sqrt{2} \mu$, from (5.40) we can estimate $T_{c r}$,

$$
T_{c r}=\frac{2 \mu}{\sqrt{\lambda}} \sim \frac{2 \mu}{g^{2}} \sim \frac{\sqrt{2} m\left(\Omega_{s}\right)}{4 \pi / 45}=5 m\left(\Omega_{s}\right) .
$$

We may take $T_{c r} \sim T\left(t_{A}\right)$, because $\left\langle\omega_{s}\right\rangle=\left\langle\omega_{v}\right\rangle=0$ when $T_{s}=T_{c r}$ and $t=t_{A}$.

The temperature will strikingly decrease in the process of inflation, but the potential energy

$V_{g, \text { eff }}\left(\varpi_{s}=\varpi_{v}=0\right)=V_{0}$ cannot decrease to $V_{g, \text { eff, min }}$ at once. This is a super-cooling process. We can get the expecting results by suitably choosing the parameters in (2.8)-(2.10). In order to estimate $H=\sqrt{\eta V_{0}}$, taking $V_{0} \sim \mu^{4} / 4 \lambda$, from (7.8) we have

$$
H=a T_{c r}^{2}, a \equiv \sqrt{\eta \lambda} / 8 \sim g^{2} \sqrt{\eta} / 8 .
$$

$T_{c r}$ is larger than the temperature corresponding to GUT. Taking $T_{c r} \sim 5 m\left(\Omega_{s}\right) \sim 5 \times 10^{15} \mathrm{Gev}$ and $\sqrt{\lambda} / 8 \sim g^{2} \sim 0.035$, we have $H^{-1}=10^{-35}$ s. If the duration of the super-cooling state is $10^{-33} \mathrm{~s}, R_{c r}$ will increase $e^{100} \sim 10^{43}$ times. The result is consistent with the Guth's inflation model [28].

If there is no $v$-matter, because of contraction by gravitation, the world would become a thermal-equilibrating singular point, i.e., the world would be in the hot death state. As seen, it is necessary that there are both s-matter and v-matter and both the S-breaking and the V-breaking.

\section{Evolving Process of Space after Inflation}

\subsection{The Reheating Process}

After inflation, the temperature must sharply descend. In this case, it is easily seen that the most symmetric state with $\left\langle\omega_{s}\right\rangle=\left\langle\omega_{v}\right\rangle=0$ is no longer stable and must decay into such a state with $V_{\min }$. This is the reheating process. Either of the $S$-breaking and the $V$-breaking can come into being, because s-matter and v-matter are completely symmetric at $t \sim t_{A}$. Letting the $V$-breaking comes into being, then the symmetry breaking is $S U_{V}(5) \rightarrow S U_{V}(3) \times S U_{V}(2) \times U_{V}(1) \rightarrow S U_{V}(3) \times U_{V}(1)$ and $S U_{S}(5)$ symmetry is still kept all time. After the reheating process, when temperature is low, considering (3.15) we have

$$
\begin{aligned}
& \varpi_{s}=0, \varpi_{v}=\varpi_{v 0}, V_{s}\left(\varpi_{s}\right)=0, V_{g}=-V_{1}, \\
& \Delta V=V_{v}(0,0)-V_{v}\left(\varpi_{v 0}\right)=V_{0}+V_{1} .
\end{aligned}
$$

After reheating, $\Delta V$ must first transform into v-energy by (2.9) and the $S U_{V}(5)$ couplings and into s-energy by (2.10). Letting $\kappa \Delta V$ transform the v-energy, then $(1-\kappa) \Delta V$ transforms the s-energy. It is necessary $\kappa>(1-\kappa)$. There is $\rho_{v}^{\prime}=\rho_{s}^{\prime}$ before the reheating. Thus, after reheating, we have

$$
\rho_{v}=\rho_{v}^{\prime}+\kappa \Delta V>\rho_{s}=\rho_{s}^{\prime}+(1-\kappa) \Delta V
$$

\subsection{The Change of Mass Densities}

Let $T_{p l}$ be such a temperature that particles exist in the $S U(5)$ plasma form when $T \geq T_{p l}$, and particles exist in the form of $S U(5)$ color singlets when $T<T_{p l}$. After reheating process, in the initial stage, both $T_{v}$ and $T_{s}$ are high and all particles must exist in the plasma form when $T_{v}>T_{s} \geq T_{p l}$. Thus, the masses of particles may be neglected so that $\rho_{s} \sim \rho_{v} \propto R^{-4}$. After temperature descends further so that $T_{s}<T_{p l}$, s-particles will form $S U_{S}(5)$ color singlets whose masses are all non-zero. Thus, there is no s-photon, i.e. 
$\rho_{s \gamma}=0$. The $S U_{S}(5)$ color singlets cannot form any clustering and their masses are all small. Let $m_{c s}$ is the largest mass of the stable $S U(5)$ color singlets, then we may suppose $m_{c s} \sim m_{p}$, here $m_{p}$ is the mass of a proton. However $v$-particles will exist in the forms of nucleons, leptons and photons, and can form galaxies in low temperatures. Consequently, in the $V$-breaking,

$$
\rho_{s}=\rho_{s m}=\rho_{s M}+\rho_{s l}, \quad \rho_{v}=\rho_{v M}+\rho_{v l}+\rho_{v y}
$$

Let the reheating process ends at $t_{r e}$. Considering $\rho_{v y}\left(t_{r e}\right)>\rho_{v m}=\rho_{v M}+\rho_{v l}$, we suppose $\rho_{s m}>\rho_{v m}$. From (8.3) we have

$$
\rho_{v m}\left(t_{r e}\right)+\rho_{v y}\left(t_{r e}\right)>\rho_{s m}\left(t_{r e}\right)>\rho_{v m}\left(t_{r e}\right) .
$$

After reheating process ends, temperature is low, $\dot{V}_{g, \text { eff }}=0$ and all masses of the Higgs particles are large enough so that the transformation $\rho_{v}$ and $\rho_{s}$ may be neglected. Thus, $\dot{\rho}_{g, t r}=0$.

As mentioned in section 4 (see (4.21)-(4.26)), the evolving laws of $\rho_{M}, \rho_{m}$ and $\rho_{\gamma}$ as space contraction are different from each other. For simplicity, we do not differentiate $\rho_{M}$ and $\rho_{m}$ for a time. Thus, neglecting $\rho_{m}$ and $\rho_{M}$, considering $p_{v \gamma}=\rho_{v \gamma} / 3$ and $\rho_{s \gamma}=0$ in the $V$-breaking, from (4.24)-(4.26) $(s \rightarrow v$ in (4.26)) and (8.5) we have

$$
\begin{aligned}
& \rho_{m g} R^{3}=\left(\rho_{v m}-\rho_{s m}\right) R^{3}=-C_{m g}=\rho_{m g 0} R_{0}^{3}<0, \\
& \rho_{s \gamma g} R^{4}=\rho_{s \gamma} R^{4}=C_{\gamma g}=\rho_{s \gamma 0} R_{0}^{4}>0,
\end{aligned}
$$

where both $C_{m g}$ and $C_{\gamma g}$ are constants. From (4.8), (8.3) and (8.6)-(8.7), (4.5)-(4.6) is reduced to

$$
\begin{aligned}
& \dot{R}^{2}=1+\eta\left(-\frac{C_{m g}}{R}+\frac{C_{\gamma g}}{R^{2}}-V_{1}\right), \\
& \ddot{R}=\frac{\eta}{2}\left(\frac{C_{m g}}{R^{2}}-2 \frac{C_{\gamma g}}{R^{3}}-V_{1}\right) .
\end{aligned}
$$

As mentioned in section 3, $V_{1}$ may be neglected when $\rho_{v}>0$ in the $V$-breaking. Thus we neglects $V_{1}$ for a time in the following.

We discuss (8.8)-(8.9) as follows.

1. If $1-\eta C_{m g}^{2} / 4 C_{\gamma g}>0$, when $R<R_{1} \equiv 2 C_{\gamma g} / C_{m g}, \ddot{R}<0$ and $\dot{R}>0$, i.e. space expands with a deceleration; when $R=R_{1}, \quad \ddot{R}=0$ and $\dot{R}=\dot{R}_{\min }=\left(1-\eta C_{m g}^{2} / 4 C_{\gamma g}\right)^{1 / 2}>0$; when $R>R_{1}, \quad \ddot{R}>0$ and $\dot{R}>0$ i.e. space expands with an acceleration. In the process, $\ddot{R}$ increases from $\ddot{R}=0$ to $\ddot{R}_{\max }=\eta C_{m g}^{3} / 54 D_{\gamma g}^{2}$ when $R=3 D_{\gamma g} / C_{m g}$, then $\ddot{R}$ decreases from $\ddot{R}_{\max }$ to $\ddot{R}_{0}>0$.

2. If $1-\eta C_{m g}^{2} / 4 C_{\gamma g}=0$, when $R<R_{1} \equiv 2 C_{\gamma g} / C_{m g}, \ddot{R}<0$ and $\dot{R}>0$; when $R=R_{1}, \ddot{R}=\dot{R}=0$; In the case, space can be static.

3. If $1-\eta C_{m g}^{2} / 4 C_{\gamma g}<0$, when $R<R_{2} \equiv\left(\eta C_{m g} / 2\right)\left[1-\sqrt{1-4 C_{\gamma g} / \eta C_{m g}^{2}}\right], \ddot{R}<0$ and $\dot{R}>0$; when $R=$ $R_{2}, \quad \ddot{R}<0$ and $\dot{R}=0$. In the case, space will begin to contract.

The first case is consistent with observations. A computation in detail is the same as that of Ref. [10].

Even $\chi_{s}$ and $\chi_{v}$ are considered, the above conclusions still hold qualitatively.

\subsection{To Determine $a(t)$}

Letting $\quad a=R / R_{0}, \quad \dot{a}_{0}^{2}=H_{0}^{2} \equiv \eta \rho_{g c}, \quad \Omega_{g m 0}=\left(\rho_{s m 0}-\rho_{v m 0}\right) / \rho_{g c}, \quad \Omega_{v \gamma 0}=\rho_{v \gamma 0} / \rho_{g c} \quad$ and $\quad \Omega_{g 0}=\Omega_{g m 0}-\Omega_{v \gamma 0}$, and considering

$$
\begin{aligned}
& \rho_{s m}-\rho_{v m}-\rho_{v \gamma}=\rho_{g c}\left[\Omega_{g m 0} / a^{3}-\Omega_{v \gamma 0} / a^{4}\right], \\
& H_{0}^{2}\left(1+\Omega g_{0}\right)=1 / R_{0}^{2}, k=-1,
\end{aligned}
$$

we rewrite (8.6) as 


$$
\dot{a}^{2}=H_{0}^{2}\left(1+\Omega_{g 0}\right) \cdot\left[1-\frac{1}{\left(1+\Omega_{g 0}\right)}\left(\frac{\Omega_{g m 0}}{a}-\frac{\Omega_{v \gamma 0}}{a^{2}}\right)\right] .
$$

From (8.12) we have

$$
t=t_{0}-\frac{1}{H_{0} \sqrt{1+\Omega_{g 0}}}\left\{\sqrt{1-M+\Gamma}-\sqrt{a^{2}-M a+\Gamma}+\frac{M}{2} \ln \frac{2-M+2 \sqrt{1-M+\Gamma}}{2 a-M+2 \sqrt{a^{2}-M a+\Gamma}}\right\},
$$

If $t_{0}$ is taken as

$$
\begin{aligned}
& t_{0}=\frac{1}{H_{0} \sqrt{1+\Omega_{g 0}}}\left\{1-M+\Gamma+\frac{M}{2} \ln \left[1-\frac{2}{M}-\frac{2 \sqrt{1-M+\Gamma}}{M}\right]\right\}, \\
& t=\frac{1}{H_{0} \sqrt{1+\Omega_{g 0}}}\left\{\sqrt{a^{2}-M a+\Gamma}+\frac{M}{2} \ln \left[1-\frac{2 a}{M}-\frac{2 \sqrt{a^{2}-M a+\Gamma}}{M}\right]\right\},
\end{aligned}
$$

where $M=\Omega_{g m 0} /\left(1+\Omega_{g 0}\right)$, and $\Gamma=\Omega_{v y 0} /\left(1+\Omega_{g 0}\right)$.

Taking $\Omega_{v \gamma 0}=0.001, \quad \Omega_{g m 0}=0.3 \Omega_{v \gamma 0}+2 \sqrt{\Omega_{v \gamma 0}}, \quad H_{0}^{-1}=9.7776 \times 10^{9} h^{-1} \mathrm{yr}$ [18]. and $h=0.8$, we get $a(t)$.

$a(t)$ is shown by the curve $A$ in the Figure 2 and describes evolution of the universe from $14 \times 10^{9} \mathrm{yr}$ ago to now. Taking $\Omega_{v \gamma 0}=0.05, \Omega_{g m 0}=2 \sqrt{\Omega_{v \gamma 0}}$, we get the $a(t)$ which is shown by the curve $B$ in the Figure 2 and describes evolution of the cosmos from $13.7 \times 10^{9}$ yr ago to now. Provided $2\left(\Omega_{v \gamma 0}+\sqrt{\Omega_{v \gamma 0}}\right)>\Omega_{g m 0}$ which is equivalent to $1-\eta C_{m g}^{2} / 4 C_{\gamma g}>0$, we can get a curve of $a(t)$ which describes evolution of the cosmological scale. The curves $A$ and $B$ show that when the parameters alter in a definte scope, the qualitative features of the evolving curves are changeless, but their concrete-changing forms are differnt from each other. Thus, the parameters in the model should be determined based on astronomical observations.

From the two curves we see that the cosmos must undergo a period in which space expands with a deceleration in the past, and undergo the present period in which space expands with an acceleration.

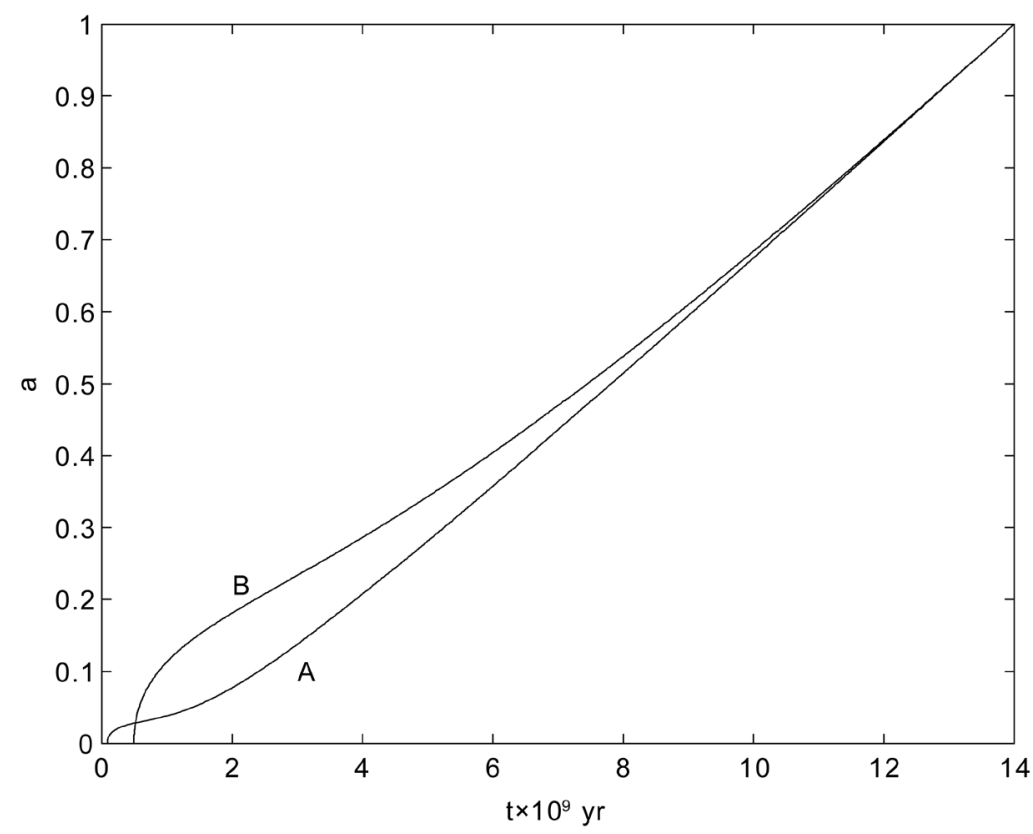

Figure 2. The curve $A$ describes evolution of $a(t)$ from $14 \times 10^{9}$ yr ago to now; The curve $B$ describes evolution of $a(t)$ from $13.7 \times 10^{9}$ yr ago to now. The starting point of curve $A$ is different from that of curve $B$. 
It should be noted that $\rho_{g 0}=\rho_{v 0}-\rho_{s 0}$ in the V-breaking, but here $\Omega_{g m 0}=-\rho_{g 0} / \rho_{c}$. Neglected $\Omega_{v \gamma 0}$, noting $k=-1$ and taking

$$
\begin{gathered}
\sqrt{1-M}=\frac{1}{\sqrt{1+\Omega_{m g}}}, M=\frac{\Omega_{m g}}{1+\Omega_{m g}}, \\
\sqrt{a^{2}-M a}=\frac{\sqrt{1-z \Omega_{m g}}}{(1+z) \sqrt{1+\Omega_{m g}}}, a=\frac{1}{1+z}, \\
t_{0}=\frac{1}{H_{0}\left(1+\Omega_{m g}\right)}\left[1+\frac{\Omega_{m g}}{2\left(1+\Omega_{m g}\right)^{1 / 2}} \ln \frac{2+\Omega_{m g}+2 \sqrt{1+\Omega_{m g}}}{-\Omega_{m g}}\right],
\end{gathered}
$$

we can reduce (8.15) to

$$
t=\frac{1}{H_{0}\left(1+\Omega_{m g}\right)}\left\{\frac{\sqrt{1-z \Omega_{m g}}}{1+z}+\frac{\Omega_{m g}}{2 \sqrt{\left(1+\Omega_{m g}\right)}} \ln \frac{2+(1-z) \Omega_{m g}+2 \sqrt{\left(1+\Omega_{m g}\right)\left(1-z \Omega_{m g}\right)}}{(1+z)\left(-\Omega_{m g}\right)}\right\} .
$$

Replacing $\Omega_{m g}$ by $\left(-\Omega_{g m 0}\right)$ because $\Omega_{g m 0}=-\rho_{g 0} / \rho_{c}$ and and ignoring $\Omega_{v \gamma 0}$, we see (8.19) to be the same as the corresponding formula (3.44) in Ref. [18].

\subsection{The Relation between Redshift and Luminosity Distance}

From (8.12) and the $R W$ metric we have

$$
\begin{aligned}
H_{0} d_{L}= & H_{0} R_{0} r(1+z)=\frac{\int_{a}^{1} \frac{c \mathrm{~d} a}{R \dot{a}}=-\int_{r}^{0} \frac{\mathrm{d} r}{\sqrt{1+r^{2}}},}{\left(\Omega_{g m 0}-2 \Omega_{v \gamma 0}\right)^{2}-4 \Omega_{v \gamma 0}} \cdot\left\{2\left(1+\Omega_{g 0}\right)-(1+z) \Omega_{g m 0}\right. \\
& \left.-\left[2\left(1+\Omega_{g 0}\right)-\Omega_{g m 0}\right] \cdot \sqrt{1-\left(\Omega_{g m 0}-2 \Omega_{v \gamma 0}\right) z+\Omega_{v \gamma 0}^{2} z^{2}}\right\},
\end{aligned}
$$

where $z=(1 / a)-1$ is the redshift caused by $R$ increasing.

Considering $\Omega_{g m 0}$ in (8.21) corresponds to $\left(-\Omega_{m 0}\right)$ in (3.81) in Ref. [18] and $\Omega_{g 0}=\Omega_{g m 0}-\Omega_{v \gamma 0}$, we see that (8.21) is consistent with (3.81) in Ref. [18].

Ignoring $\Omega_{v y 0}$, taking $\Omega_{g m 0} \rightarrow\left(-\Omega_{g m 0}\right)$, we reduce (8.21) to

$$
H_{0} d_{L}=\frac{2 c}{\Omega_{g m 0}^{2}}\left\{2+\Omega_{g m 0}(1-z)-\left(2+\Omega_{g m 0}\right) \sqrt{1-\Omega_{g m 0} z}\right\},
$$

which is consistent with (3.78) in Ref. [18]. Approximating to $\Omega_{m 0}^{1}$ and $z^{2}$, we obtain

$$
H_{0} d_{L}=z+\frac{1}{2} z^{2}\left(1+\frac{1}{2} \Omega_{g m 0}\right)
$$

Taking $\Omega_{v \gamma 0}=0.001, \Omega_{g m 0}=0.3 \Omega_{v \gamma 0}+2 \sqrt{\Omega_{v \gamma 0}}$ and $H_{0}^{-1}=9.7776 \times 10^{9} h^{-1}$ yr [18] and $h=0.8$, from (8.22) we get the $d_{L}-z$ relation which is shown by the curve $A$ in the Figure 3. Taking $\Omega_{v \gamma 0}=0.05$ and $\Omega_{g m 0}=2 \sqrt{\Omega_{v \gamma 0}}$, we get the $d_{L}-z$ relation which is shown by the curve $B$ in the Figure 3 .

\section{After Expansion with an Acceleration, Space Expands with a Deceleration, Then Comes to Static and Finally Begin to Contract.}

As mentioned before, the evolving laws of $\rho_{M}$ and $\rho_{m}$ as space contracts or expands are different from each other. After space expands with an acceleration, we should consider the difference between $\rho_{M}$ and $\rho_{m}$. 


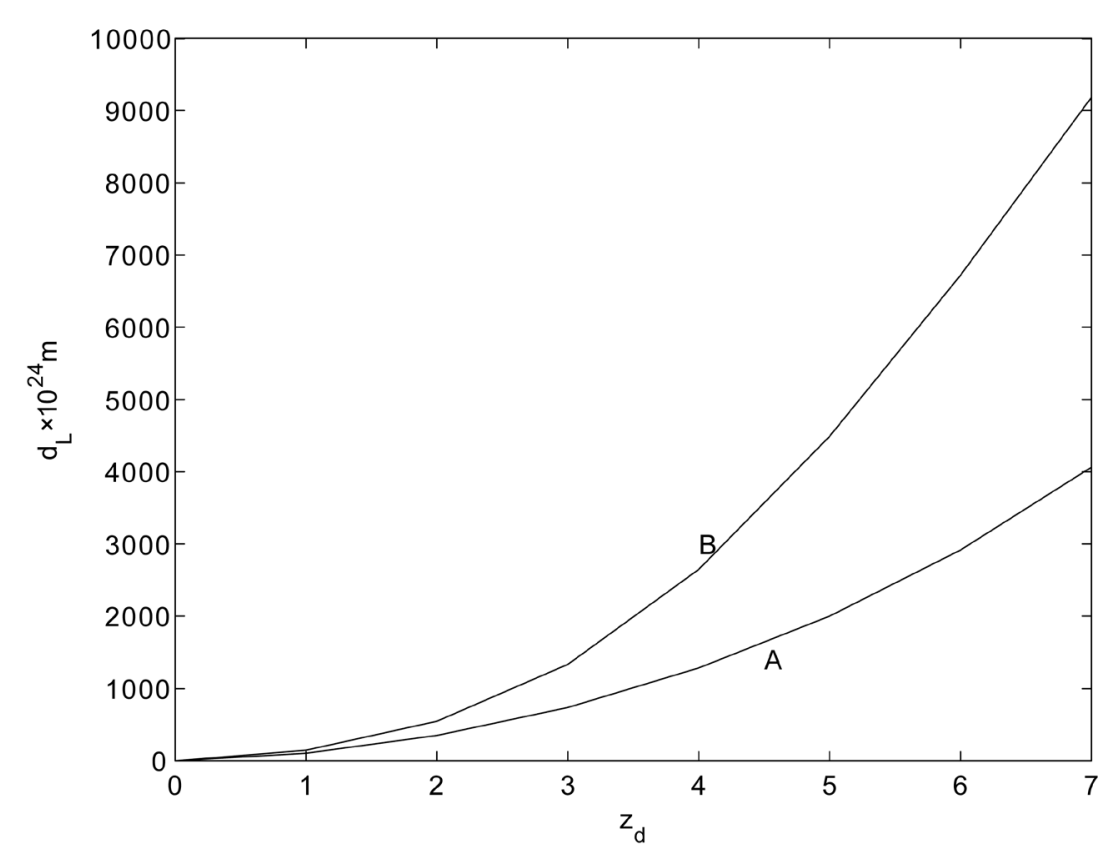

Figure 3. The curve A describes the $d_{L}-z$ relation when $\Omega_{v y 0}=0.001$ and $\Omega_{m 0}=0.3 \Omega_{v \gamma 0}+2 \sqrt{\Omega_{v y 0}}$; The curve B describes the $d_{L}-z$ relation when $\Omega_{v \gamma 0}=0.05$ and $\Omega_{m 0}=2 \sqrt{\Omega_{v \gamma 0}}$.

When $R$ is large enough, $\rho_{g \gamma}=\rho_{v \gamma} \ll \rho_{v l} \ll \rho_{v M}$ so that $\rho_{g \gamma}$ and $\rho_{v l}$ may be neglected. Thus, considering $\dot{V}_{g}=0$ in low temperatures, neglecting $\rho_{v m}$ and $\rho_{v y}$, we can reduce (4.5)-(4.6) to

$$
\begin{aligned}
& \dot{R}^{2}=1+\eta\left(\frac{C_{v M}}{R}-\frac{C_{s m}}{R^{1+3 l_{s}}}-V_{1} R^{2}\right), \\
& \ddot{R}=-\frac{\eta}{2}\left(\frac{C_{v m}}{R}-\frac{C_{s m}}{R^{1+3 l_{s}}}+2 V_{1} R\right) .
\end{aligned}
$$

where $C_{v M} \equiv \rho_{v M 0} R_{0}^{3}, \quad C_{s m} \equiv \rho_{s m 0} R_{0}^{3\left(1+l_{s}\right)}$ and $C_{s m}>C_{v M}$. As mentioned in section $3, V_{1}$ is so small that it may be neglected when $\rho_{v}>0$ in the $V$-breaking. It is seen from (9.2) that $\ddot{R}$ changes from $\ddot{R}>0$ to $\ddot{R}=0$ and finally $\ddot{R}<0$ as space expands. It is seen from (9.1) that there must be $R_{\max }$ so that $\dot{R}_{\max }=0$ when $t=t_{\max }$. Space will begin to contract when $t>t_{\max }$, because $\dot{R}_{\max }=0$ and $\ddot{R}<0$ at $t_{\max }$. It is seen that after space expands with an acceleration, it will expands with a deceleration, then comes to static, and final begin contracts. This is different from the conventional theory and the model [19] [20].

When $R$ is large enough, $C_{v M} / R$ and $C_{s m} / R^{2}$ may be neglected. Thus, from (9.1) we have

$$
R_{\max }=\sqrt{1 / \eta V_{1}} \text {. }
$$

To sum up, according to the present model, the universe can expand from $R_{\min }$ to $R_{\max }$, and then contract to $R_{\min }$; Both $R_{\max }$ and $R_{\min }$ are finite. The universe can be in the $S$-breaking, and can be in the $V$-breaking as well; The $S$-breaking can transform to the $V$-breaking after space contracts to $R_{\min }$ and vice versa.

\section{Existing and Distribution Forms of $S U_{S}(5)$ Color Singlets}

In the V-breaking, all s-gauge particles and s-fermions are massless. When the temperature $T_{s} \geq T_{p l}$, all $s$-particles must exist in plasma form. When $T_{s}<T_{p l}$, all s-particles will exist in $s-S U(5)$ color-singlets (conjecture 2). Let $A, B, C, D, E$ denote the 5 sorts of colors. A component of 10 representation carries color $\alpha \beta, \alpha, \beta=A, B, C, D, E, \alpha \neq \beta$. A component of $\underline{5}$ representation carries color $\alpha$. A gauge 
boson carries colors $\alpha \beta^{*}$. There are the following sorts of the s-SU(5) color-singlets.

2-fermion states: $\alpha \alpha^{*}$ or $(\alpha \beta)(\alpha \beta)^{*}, \alpha \neq \beta$. 3-fermion states: $(\underline{A B})(\underline{C D}) \underline{E}$ or $(\underline{A B}) \underline{A^{*}} \underline{B}^{*}$. 4-fermion states: $(\underline{A B}) \underline{C D E}$. 5-fermion states: $\underline{A B C} \underline{D E} \underline{E}$ or $(\underline{A B})(\underline{B C})(\underline{C D})(\underline{D E})(\underline{E A})$. Gauge boson single-states: $\left(\underline{\alpha \beta^{*}}\right)\left(\underline{\alpha^{*} \beta}\right)$ or $\left(\underline{\alpha \beta^{*}}\right)\left(\underline{\beta \gamma^{*}}\right)\left(\underline{\gamma \alpha^{*}}\right)$ etc., $\alpha \neq \beta \neq \gamma$. Fermion-gauge boson singlets: $\underline{\alpha}\left(\underline{\alpha \beta^{*}}\right) \underline{\beta}$, $\underline{\alpha^{*}}\left(\left(\alpha \gamma^{*}\right)\left(\gamma \beta^{*}\right)\right) \underline{\beta}$ etc.

The masses of all color singlets are non-zero, hence $\rho_{s \gamma}=0$. The fermions with the spin $s=1 / 2$ and the least mass are stable, and the bosons with the spin $s=0$ and the least mass are stable as well. This is because there is no the electroweak interaction among $S U(5)$ color single states so that they cannot decay. Of course, there are the s-antiparticles corresponding to the s-colour singlets above as well.

There is no interaction among the $S U_{S}(5)$ color singlets, because $S U(5)$ is a simple group. There are interaction among the $S U_{S}(5)$ color singlets by exchanging the $S U_{S}(5)$ color single states. The interaction radius must be very small because the masses of all $S U_{S}(5)$ color singlets are non-zero. Thus, the interaction may be neglected so that we can approximately regard the $S U_{S}(5)$ color singlets as ideal gas without collision. The ideal gas has the effect of free flux damping for clustering. Consequently, the $S U_{S}(5)$ color single states cannot form clustering and must distribute loosely in space, and their decoupling temperature must be very high so that their relative velocities are large and invariant. But they can form s-superclusterings, because there is the gravitation among them and there is repulsion between s-matter and v-matter. The superclusterings are similar to neutrino superclusterings and are huge voids for $v$-observers.

\section{New Predictions, an Inference, and There Is No Restriction for $T^{\mu \nu}$}

\subsection{New Predictions}

\subsubsection{The Essence and Characters of Huge Voids}

It is possible that Huge voids are not empty and are equivalent to huge concave lenses. The density of hydrogen inside the huge voids is more less than that predicted by the conventional theory.

Based on above mentioned, we consider, the huge voids for the v-observers are, in fact, superclusters of the $S U_{S}(5)$ color singlets. The huge $v$-voids are not empty. There must be the $S U_{S}(5)$ color singlets inside them, and $\rho_{s}^{\prime} \gg \rho_{v}^{\prime}, \rho_{s}^{\prime}>\rho_{s}$, and $\rho_{v}^{\prime}<\rho_{v}$. Here $\rho_{s}^{\prime}$ and $\rho_{v}^{\prime}$ denote the densities inside the huge $v$-voids, and $\rho_{s}$ and $\rho_{v}$ denote the densities outside the huge $v$-voids. The characters of such a huge $v$-void are as follows:

A. A $v$-void must be huge, because there is no other interaction among the $s$-SU(5) color singlets except the gravitation and the masses of the $s-S U(5)$ color singlets are very small.

B. When $v$-photons pass through such a huge $v$-void, the $v$-photons must suffer repulsion coming from $s$-matter inside the huge void and are scattered by the $v$-void as they pass through a huge concave lens. Consequently, the galaxies behind the huge $v$-void seem to be darker and more remote. Hence the huge voids are equivalent to huge concave lenses.

C. Both density of matter and density of dark matter in the huge voids must be more lower than those predicted by the conventional theory. Consequently, the densities of hydrogen and helium inside the huge voids must be more less than that predicted by the conventional theory.

The predict can be confirmed or negated by the observation of hydrogen distribution.

This is a decisive prediction which distinguishes the present model from other models.

\subsubsection{The Gravity between Two Galaxies Whose Distance Is Long Enough}

There must be s-superclusterings between two v-galaxies when both distance is long enough, hence the gravity between the two v-galaxies must be less than that predicted by the conventional theory due to the repulsion between s-matter and v-matter. When the distance between two v-galaxies is small, the gravitation is not influenced by s-matter, because $\rho_{s}$ must be small when $\rho_{v}$ is big.

\subsubsection{A Black Hole with Its Mass and Density Big Enough Will Transform into a White Hole}

Letting there be a $v$-black hole with its mass and density to be so big that its temperature can arrive at $T_{v} \gtrsim T_{c r}=2 \mu / \sqrt{\lambda}$ because the black hole contracts by its self-gravitation, then the expectation values of the Higgs fields inside the v-black hole will change from $\varpi_{v}=\varpi_{v 0}$ and $\varpi_{s}=0$ into $\varpi_{v}=\varpi_{s}=0$. Consequently, 
inflation must occur. After inflation, the most symmetric state $\left(\varpi_{v}=\varpi_{s}=0\right)$ will transit into the $V$-breaking. Thus, the energy of the black hole must transform into both the v-energy and the s-energy. Thus, a v-observer will find that the black hole disappears and a white hole appears.

In the process, a part of v-energy transforms into v-energy and the other part transforms into s-energy. A $v$-observer will consider the energy not to be conservational because he cannot detect s-matter except by repulsion. The transformation of black holes is different from the Hawking radiation. This is the transformation of the vacuum expectation values of the Higgs fields. There is no contradiction between the transformation and the Hawking radiation or another quantum effect, because both describe different processes and based on different conditions. According to the present model, there still are the Hawking radiation or other quantum effects of black holes. In fact, the universe is just a huge black hold. The universe can transform from the $S$-breaking into the $V$-breaking because of its contraction. This transformation is not quantum effects.

\subsection{An inference: $\lambda_{\text {eff }}=\lambda=0$, Although $\rho_{\text {vac }} \neq 0$}

The effective cosmological constant $\lambda_{\text {eff }}=\lambda+\rho_{\text {g,vac }}$. The conventional theory can explain evolution with a small $\lambda_{\text {eff }}$, but $\rho_{g, v a c}=\rho_{\text {vac }} \ggg \lambda_{\text {eff }}$, Consequently, the issue of the cosmological constant appears.

$\rho_{\text {vac }}=0$ can be obtained by some supersymmetric model, but it is not a necessary result. On the other hand, the particles predicted by the supersymmetric theory have not been found, although their masses are not large.

$\rho_{\text {vac }}=0$ is a necessary result of our quantum field theory without divergence [13]-[15]. In this theory, $\rho_{\text {vac }}=0$ is naturally obtained without normal order of operators, there is no divergence of loop corrections, and dark matter which can form dark galaxies is predicted [16] [17]. But the model does not explain the evolution of the universe.

As mention above, the present model can explain evolution of the universe without $\lambda_{\text {eff }}$, hence

$$
\lambda_{\text {eff }}=0 \text {. }
$$

Applying the conventional quantum field theory to the present model, we have $\rho_{v a c}=\rho_{s, v a c}+\rho_{v, v a c}$, here $\rho_{\text {vac }}$ is the energy density of the vacuum state. According to the conjecture 1 , s-particles and v-particles are symmetric. Hence both ground states must be symmetric as well. Hence

$$
\rho_{s, v a c}=\rho_{v, v a c}=\rho_{v a c} / 2 \text {. }
$$

According to conjecture $1, \rho_{g s}=-\rho_{g v}$ when $\rho_{s}=\rho_{v}$. Consequently, although

$$
\rho_{\text {vac }}=\rho_{s, \text { vac }}+\rho_{v, \text { vac }}=2 \rho_{s, \text { vac }} \gg 0
$$

we have still

$$
\begin{aligned}
& \rho_{g, v a c}=\rho_{s, v a c}-\rho_{v, v a c}=0, \\
& \lambda_{\text {eff }}=\lambda+\rho_{g, v a c}=\lambda=0 .
\end{aligned}
$$

Here $\lambda$ is the Einstein cosmological constant. This is a direct inference of the present model, and independent of a quantum field theory.

Because of (11.4), for the vacuum state in the S-breaking or the V-breaking, the Einstein field equation is reduced to

$$
R_{\mu v}-\frac{1}{2} g_{\mu \nu} R=-8 \pi G\left(T_{s, v a c, \mu v}-T_{v, v a c, \mu v}\right)=-8 \pi G\left(T_{v, v a c, \mu v}-T_{s, v a c, \mu v}\right)=0 .
$$

This is a reasonable result.

\subsection{There Is No Restriction for $T^{\mu \nu}=T_{v}^{\mu \nu}+T_{s}^{\mu v}$}

The problem of total energy conservation in the general relativity is unsolved up to now. This is because tensors at different points cannot be summed up. On the other hand, according to the Einstein equation, $T_{; \mu}^{\mu \nu}=0$. In contrast with this result, according to the present model, we have (2.22). This result (2.22) implies that there is no restriction for $T_{; \mu}^{\mu \nu}=T_{s ; \mu}^{\mu v}+T_{v ; \mu}^{\mu \nu}$ or $\rho=\rho_{v}+\rho_{s}$. The dominant energy condition and the positive energy theorem are not applicable to $T^{\mu \nu}=T_{v}^{\mu \nu}+T_{s}^{\mu \nu}$.

Whether does $T_{, v}^{\mu \nu}=T_{s, v}^{\mu v}+T_{v, v}^{\mu v}+T_{g, v}^{\mu v}=0$ holds? We will discuss the problem in another paper. 


\section{Conclusions}

A new conjecture is proposed that there are s-matter and $v$-matter which are symmetric, whose gravitational masses are opposite to each other, although whose masses are all positive. Both can transform from one to another when temperature $T \sim T_{c r}$. Consequently there is no singularity in the model. The cosmological constant $\lambda_{\text {eff }}=\lambda=0$ is determined although the energy density of the vacuum state is still very large. A formula is derived which well describes the relation between a luminosity distance and the redshift corresponding to it.

The conjecture are not in contradiction with given experiments and astronomical observations up to now, although the conjecture violates the equivalence principle.

There are two sorts of breaking modes, i.e. the $S$-breaking and the V-breaking. In the V-breaking, $S U_{V}(5)$ is broken to $S U_{V}(3) \times S U_{V}(2) \times U_{V}(1)$ and finally $S U_{V}(3) \times U_{V}(1)$ and $S U_{S}(5)$ is kept all time. Consequently, $v$-particles get their masses and form $v$-atoms, $v$-observers and v-galaxies etc., while s-gauge bosons and s-fermions are still massless and must form $S U_{S}(5)$ color-single states when $T_{s}<T_{p l}$. There is no interaction among the $S U_{S}(5)$ color-single states except the gravitation, because $S U(5)$ group is a simple group. Hence they must distribute loosely in space, cannot be observed and can cause space to expand with an acceleration. Thus, $v$-matter is identified with conventional matter (include dark matter) and s-matter is similar to the dark energy. But in contrast with the dark energy, the gravitational mass of s-matter is negative in the V-breaking.

There are the critical temperature $T_{c r}$, the highest temperatures $T_{\max }$, and the least scale $R_{\min }>0$ in this model. Hence it is impossible that the Plank temperature, length and time are arrived.

Based on the present model, the space evolving process is as follows. Firstly, in the S-breaking, $\rho_{g}=\rho_{s}-\rho_{v}>0$, hence space contracts and $T_{s}$ and $T_{v}$ rises. When $T_{s} \gtrsim T_{\varphi c r}$, the transformation of $\rho_{s}$ and $\rho_{v}$ from one to another is striking so that $\rho_{g}<0$ is possible. Hence there are such solutions of the evolution equations which satisfy the physical boundary condition, i.e. $\dot{R}=0$ when $R_{\min }>0$. When $T_{s}=T_{c r}$, $\left\langle\omega_{s}\right\rangle=\left\langle\omega_{v}\right\rangle=0$, the $S U_{V}(5) \times S U_{S}(5)$ symmetry (the highest symmetry) is kept, and $\rho_{g}<0$. When space contracts further, $R$ arrives the least scale $R_{\min }>0, \quad \dot{R}=0$ and $T_{v}$ arrives the highest temperature $T_{\max } \geq T_{c r}$. Then space expands and $T_{v}$ descends to $T_{c r}$ so that $\rho_{g} \sim 0$ and inflation must occur. After the inflation, the phase transition of the vacuum (the reheating process) occurs. After the reheating process, this state with the highest symmetry transits to the state with the $V$-breaking. In the $V$-breaking, the evolving process of space is as follows. Space firstly expands with a deceleration because $\rho_{g}=\rho_{v}-\rho_{s}>0$; Secondly, space expands with an acceleration because $\rho_{g}<0$ and $k=-1$; Thirdly, space expands with a deceleration, and then comes to static; Finally, space begin contract.

It is seen that according to the present model, the universe can expand from $R_{\min }$ to $R_{\min }$, and then contract from $R_{\min }$ to $R_{\min }$; Both $R_{\min }$ and $R_{\min }$ are finite. The universe can be in the $S$-breaking, and can be in the $V$-breaking as well; The $S$-breaking can transform to the $V$-breaking after space contracts to $R_{\min }$ and vice versa.

Three new predicts have been given.

Huge $v$-voids in the V-breaking are not empty, but are superclusterings of s-particles. The huge voids are equivalent to huge concave lens. The densities of hydrogen helium in the huge voids predicted by the present model must be much less than that predicted by the conventional theory.

The gravitation between two galaxies whose distance is long enough will be less than that predicted by the conventional theory.

It is possible that a $v$-black hole with its big enough mass and density can transform into a huge white hole by its self-gravitation.

I am very grateful to professor Zhao Zhan-yue, professor Wu Zhao-yan, professor Zheng Zhi-peng and professor Zhao Zheng-guo for their helpful discussions and best support. I am very grateful to professor Liu Yun-zuo, professor Lu Jingbin, doctor Yang Dong and doctor Ma Keyan for their helpful discussions and help in the manuscript.

\section{References}

[1] Hawking, S.W. and Ellis, G.F.R. (1999) The Large Scale Structure of Space-Time, Cambridge University Press, 7, 98, 101, 137, 256-298. 
[2] Brandenberger, R., Mukhanov, V. and Sornborger, A. (1993) Cosmological Theory without Singularities. Physical Review D, 48, 1629-1642. http://dx.doi.org/10.1103/PhysRevD.48.1629

[3] Frolov, V.P, Markov M.A. and Mukhanov V.F. (1990) Black Holes as Possible Sources of Closed and Semiclosed Worlds. Physical Review D, 41, 383-394. http://dx.doi.org/10.1103/PhysRevD.41.383

[4] Caldwell, R.R. (2004) Dark Energy. Physics World, 17, 37-42.

[5] Padmanabhan, T. (2003) Cosmological Constant-The Weight of the Vacuum. Physics Reports, 380, 235-320. http://dx.doi.org/10.1016/S0370-1573(03)00120-0

[6] Peebles P.J.E. and Ratra, B. (2003) The Cosmological Constant and Dark Energy. Reviews of Modern Physics, 75, 559. http://dx.doi.org/10.1103/RevModPhys.75.559

[7] Weinberg, S. (1987) Anthropic Bound on the Cosmological Constant. Physical Review Letters, 59, 2607-2610. http://dx.doi.org/10.1103/PhysRevLett.59.2607

[8] Martel, H., Shapiro, P.R. and Weinberg, S. (1998) Likely Values of the Cosmological Constant. The Astrophysical Journal, 492, 29-40. http://dx.doi.org/10.1086/305016

[9] Peebles, P.J.E. and Ratra, B. (1988) Cosmology with a Time-Variable Cosmological “Constant”. The Astrophysical Journal, 325, L17-L20. http://dx.doi.org/10.1086/185100

[10] Ratra, B. and Peebles, P.J.E. (1988) Cosmological Consequences of a Rolling Homogeneous Scalar Field. Physical Review D, 37, 3406; http://dx.doi.org/10.1103/PhysRevD.37.3406

[11] Peebles, P.J.E. and Ratra, B. (2003) The Cosmological Constant and Dark Energy. Reviews of Modern Physics, 75, 559. http://dx.doi.org/10.1103/RevModPhys.75.559

[12] Hall, L.J. Nomura Y. and Oliver, S.J., (2005) Evolving Dark Energy with w $\neq-1$. Physical Review Letters, 95, 14. http://dx.doi.org/10.1103/PhysRevLett.95.141302

[13] Chen, S.H. (2002) Quantum Field Theory without Divergence A. arXiv: hep-th/0203220.

[14] Chen, S.H. (2002) Significance of Negative Energy State in Quantum Field Theory A. arXiv: hep-th/0203230.

[15] Chen, S.H. (2005) Quantum Field Theory without Divergence. In: Kovras, O., Ed., Quantum Field Theory: New Research, Nova Science Publishers, Hauppauge, 103-170.

[16] Chen, S.H. (2001) A Possible Candidate for Dark Matter. arXiv: hep-th/0103234.

[17] Chen, S.H. (2005) A Possible Candidate for Dark Matter. In: Val Blain, J., Progress in Dark Matter Research, Nova Science Publishers, Hauppauge, 65-72.

[18] Peacock, J.A. (1999) Cosmological Physics. Cambridge University Press, 78, 89, 90, 296, 458, 460-464, $579,664$.

[19] Chen, S.-H. (2009) Discussion of a Possible Universal Model without Singularity. arXiv: 0908.1495v2.

[20] Chen, S.-H. (2006) A Possible Universal Model without Singularity and its Explanation for Evolution of the Universe. High Energy Physics_-Phenomenology, arXiv:hep-ph/0611283.

[21] Gibbons, G.W. and Hawking, S.W. (1977) Action Integrals and Partition Functions in Quantum Gravity. Physical Review D, 15, 2752. http://dx.doi.org/10.1103/PhysRevD.15.2752

[22] Chaichian, M. and Nelipa, N.F. (1984) Introduction to Gauge Field Theories. Springer-Verlag, Berlin, Heidelberg, 269.

[23] Ross, G.G. (1984) Grand Unified Theories. Benjamin/Cummings Publishing Company, Inc., 177-183.

[24] Weinberg, S. (1972) Gravitation and Cosmology. Wiley, New York, Chapter 12, Section 3.

[25] Coleman, S. and Weiberg, E.J. (1973) Radiative Corrections as the Origin of Spontaneous Symmetry Breaking. Physical Review D, 7, 1888-1910. http://dx.doi.org/10.1103/PhysRevD.7.1888

[26] Brandenberg, R.H. (1985) Quantum Field Theory Methods and Inflationary Universe Models. Reviews of Modern Physics, 57, 1-60. http://dx.doi.org/10.1103/RevModPhys.57.1

[27] Liu, L., Jiang, Y. and Qian, Z. (1989) The Inflationary Universe Scenario in $10^{-35}$ Sec after Big-Bang. Progress in Physics, 9, 121-187. (in Chinese)

[28] Guth, A.H. (1981) Inflationary Universe: A Possible Solution to the Horizon and Flatness Problems. Physical Review D, 23, 347-356. http://dx.doi.org/10.1103/PhysRevD.23.347.53 